\title{
Secrecy Performance Analysis for TAS-MRC System with Imperfect Feedback
}

\author{
Jun Xiong, Yanqun Tang, Dongtang Ma, Pei Xiao, and Kai-Kit Wong
}

\begin{abstract}
In this paper, we investigate the secrecy performance for a multiple-input multiple-output (MIMO) wiretap channel in the presence of a multi-antenna eavesdropper. In particular, the legitimate transmitter uses transmit antenna selection (TAS) to transmit on a single antenna with the largest signal-to-noise ratio (SNR) while both the legitimate receiver and the eavesdropper adopt maximal ratio combining (MRC) for reception. We derive exact closed-form expressions for the probabilities of positive secrecy rate and secrecy outage with imperfect feedbacks due to feedback delay and/or error. Furthermore, we derive the asymptotic secrecy outage probability at high signal-to-noise ratio (SNR) for the transmitter-receiver channel, which accurately reveals the secrecy diversity loss due to imperfect feedbacks. Simulation results are provided to verify our analytical results and illustrate the impacts of imperfect feedback on the secrecy performance of such a wiretap system.
\end{abstract}

Index Terms-Antenna selection, Feedback, Physical-layer security, Secrecy outage probability, Wiretap channel.

\section{INTRODUCTION}

$\mathbf{P}$ HYSICAL-layer security (PLS) is an emerging research area that aims at developing secure communication systems by exploiting randomness properties of wireless channels. It was pioneered in the 1970 s by Wyner [1], who introduced the wiretap channel, and the result was later extended to the scalar Gaussian wiretap channels [2] and broadcast channels [3]. In [2], the secrecy capacity was defined as the maximum rate of communication such that the information can be decoded reliably at the legitimate receiver but cannot be inferred at any positive rate at the eavesdropper. Recently, security in wiretap channels with multiple antennas has been examined in several papers. With the additional spatial degrees of freedom (DoF) provided by multi-antenna systems, the limitation that the main channel (between the transmitter and the receiver) could be worse than the eavesdropper channel (between the transmitter and the eavesdropper) can be overcome. In particular, the achievable secrecy rate in the Gaussian multiple-input single-output (MISO) wiretap channel was studied in [4], [5], which demonstrated that the optimal communication strategy was beamforming (i.e., the rank of the input covariance matrix was one). Following further efforts, the secrecy capacity of

This research is supported in part by the China Natural Science Foundation under Grant 61372099 and Grant 61302140.

J. Xiong, Y. Tang, and D. Ma are with the School of Electronic Science and Engineering, National University of Defense Technology, Changsha, 410073, China (E-mails: $\{x j 8765$, tangyanqun, dongtangma $\} @$ nudt.edu.cn).

P. Xiao is with Center for Communication Systems Research, University of Surrey, UK (E-mail: p.xiao@surrey.ac.uk).

$\mathrm{K}$. K. Wong is with the Department of Electronic and Electrical Engineering, University College London, UK (E-mail: kai-kit.wong@ucl.ac.uk). the Gaussian multiple-input multiple-output (MIMO) wiretap channel has been fully characterized in [6]-[8].

Achieving the secrecy capacity would require the transmitter to have precise knowledge of the eavesdropper channel [4][8], which in many cases is infeasible. In this respect, artificial noise (AN) assisted beamforming, or masked beamforming, becomes a practical method of providing secure communications without the knowledge of the eavesdropper channel [9]. In masked beamforming, the transmitter allocates a fraction of its power to confuse the eavesdropper in addition to transmitting the information-bearing signal [10]. However, imprecise knowledge of the main channel will cause interference leakage to the legitimate receiver when $\mathrm{AN}$ is used. In order to address this problem, a robust beamforming was proposed in [11] to minimize the transmit power for guaranteeing a prespecified signal-to-interference-plus-noise ratio (SINR) for the legitimate receiver. In addition, instead of full channel state information (CSI) of the main channel back to the transmitter, [12] analyzed the secrecy performance of codebook beamforming transmission based on limited CSI feedback.

Although these beamforming or precoding schemes in [9][12] lessen the requirement of the knowledge of the eavesdropper channel, the problem of requiring the entire or partial CSI of the main channel at the transmitter (e.g., full CSI for masked beamforming [9], [10] and the channel estimate for robust beamforming [11]) remains, which incurs high feedback overhead from the legitimate receiver to the transmitter. On the other hand, the front-end architecture and radio frequency (RF) section of multi-antenna nodes are highly complex and expensive. One effective remedy is to use transmit antenna selection (TAS) and/or maximal-ratio combining (MRC) reception, which offers a good trade-off between complexity and performance [13]. The TAS scheme requires only a single RF chain to reduce cost, complexity and size considerably. Furthermore, only $\left\lceil\log N_{t}\right\rceil$ bits are necessary to be fed back from the legitimate receiver to the transmitter.

Without secrecy, the advantage of spatial diversity for TASMRC schemes has been well understood in [14]-[16], where the receiver selects the transmit antenna according to a certain criterion (e.g., maximizing the received signal-to-noise ratio (SNR)), and the optimal antenna index is then fed back to the transmitter. In practice, the feedback may be outdated and/or in error. The effects of time-delayed feedbacks on TASMRC schemes were investigated in [14], [15]. The results were recently extended to Nakagami- $m$ fading channels in [16] and closed-form symbol error rate (SER) expressions were derived.

In recent years, there has also been great interest on TAS$\mathrm{MRC}$ for secure communications in the presence of a single- 
or multi-antenna eavesdropper [17]-[24]. In [17], the secrecy outage probability for MRC and selection combining (SC) at the eavesdropper was compared. It was shown that SC at one multi-antenna eavesdropper will have the same effect as that at multiple non-colluding single-antenna eavesdroppers. Moreover, [18] considered MRC at both the legitimate receiver and the eavesdropper, and derived closed-form expressions for the probability of non-zero secrecy rate and the secrecy outage probability. The impact of correlation for a single-input multiple-output (SIMO) system using MRC was analyzed in [19]. Results illustrate that antenna correlation at the receiver will severely degrade the secrecy performance, especially in the low average channel gain regime. In addition, the secrecy performance for MIMO wiretap channels with orthogonal space-time block codes (OSTBCs) and arbitrary antenna correlation was also analyzed in [20]. In [21], [22], TAS was considered to improve the secrecy performance over Rayleigh and Nakagami- $m$ fading channels, respectively. Both MRC and $\mathrm{SC}$ at both the legitimate receiver and the eavesdropper were studied in [22]. The derived asymptotic secrecy outage probability indicated that the secrecy diversity order was the same as that without secrecy and independent of the number of the eavesdropper's antennas. [23] examined the impact of antenna correlation for MIMO wiretap channels in which the transmitter used TAS while both the legitimate receiver and eavesdropper used MRC. More recently, [24] proposed the TAS at the transmitter and the generalized selection combining (GSC) at the receiver to enhance secure communications.

The operation of TAS requires feedback from the receiver to the transmitter but the limitation of the analysis in [17][24] is that such feedback is assumed delay- and error-free, as such, the transmitter can always select the optimal antenna. However, perfect feedback is impossible in practical systems, and the feedback is usually imperfect [14]-[16]. In this paper, we study the impact of imperfect feedbacks on the secrecy performance for the MIMO wiretap channel in the presence of a multi-antenna eavesdropper, in which, similar to [23], the transmitter selects a single antenna to maximize the SNR at the receiver, and both the receiver and the eavesdropper employ MRC. Our main contributions are summarized as follows:

- Without secrecy, the SER for the TAS-MRC scheme with imperfect feedbacks has been well studied in [16]. The effect of outdated CSI on the secrecy outage probability for a MISO wiretap channel in the presence of a singleantenna eavesdropper with TAS scheme was also investigated recently in [25], where the exact and asymptotic expressions for secrecy outage probability were derived. Nevertheless, to the best of our knowledge, the effect of imperfect feedbacks on the secrecy performance for the TAS-MRC scheme in a general MIMO wiretap channel has not been addressed in the literature. Following the similar line of [16], our work in this paper fills this gap.

- We derive new closed-form expressions for the probability of non-zero secrecy rate and the secrecy outage probability when separately and jointly considering feedback delay and feedback errors. Our results encompass existing results with perfect feedback as special cases.

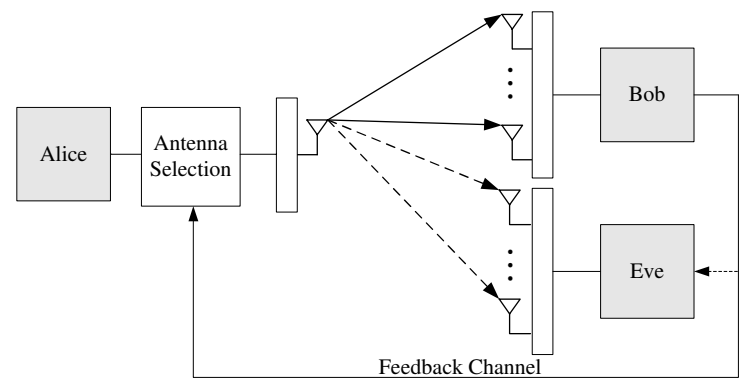

Fig. 1. A TAS system in the presence of a multi-antenna eavesdropper.

- Further, we provide asymptotic analysis for the secrecy outage probability at high SNRs for the main channel. It is demonstrated that with perfect feedbacks, the TASMRC scheme can achieve the same secrecy diversity order as that in the non-security setting, i.e., $N_{t} N_{r}$, where $N_{t}$ and $N_{r}$ denote the numbers of transmit antennas and receive antennas, respectively. However, when the feedbacks are outdated and/or in error, the diversity gain from TAS will disappear and only the MRC diversity at the receiver side can be realized, i.e., the secrecy diversity order becomes $N_{r}$. Interestingly, it can be found that the secrecy diversity order is independent of the number of the eavesdropper's antennas $N_{e}$, which only affects the secrecy array gain.

The remainder of this paper is organized as follows. Section II describes the system model of the TAS-MRC MIMO wiretap channel. In Section III, we study the effect of time-delayed feedbacks while Section IV investigates the secrecy performance with erroneous feedbacks. We characterize the secrecy performance when both feedback delays and feedback errors are present in Section V. Simulation results are presented in Section VI, and we conclude the paper in Section VII.

Notations-Upper case and lower case bold letters denote matrices and column vectors, respectively. $\mathbb{E}\{\cdot\}$ denotes statistical expectation while $\operatorname{Pr}\{\cdot\}$ denotes the probability of an input event. Also, $\|\mathbf{a}\|$ returns the Frobenious norm of vector a. We use $\mathbf{I}_{N}$ to denote an $N \times N$ identity matrix. The set of all $N$-dimensional complex vectors is denoted by $\mathbb{C}^{N}$, and $\mathbf{x} \sim \mathcal{C N}\left(\mathbf{0}, \sigma^{2} \mathbf{I}_{N}\right)$ means that $\mathbf{x}$ is a random vector following a complex circular Gaussian distribution with zero mean and covariance $\sigma^{2} \mathbf{I}_{N}$. $\lceil x\rceil$ denotes the closest integer not less than $x$ and all logarithms are base- 2 unless otherwise indicated.

\section{SYSTEM MODEL AND PRELIMINARIES}

\section{A. System model}

As shown in Fig. 1, we consider a MIMO wiretap channel with an $N_{t}$-antenna transmitter (named Alice), an $N_{r}$-antenna intended receiver (Bob), and also an $N_{e}$-antenna eavesdropper (Eve). We denote the main channel and the eavesdropper channel, respectively, by $\mathbf{H} \in \mathbb{C}^{N_{r} \times N_{t}}$, and $\mathbf{G} \in \mathbb{C}^{N_{e} \times N_{t}}$, whose elements are independent and identically distributed (i.i.d.) complex Gaussian random variables. Furthermore, the main channel and the eavesdropper channel are independent of each other. 
We assume that the CSI of the eavesdropper channel is not available to both Alice and Bob, i.e., a passive eavesdropper. The overall TAS-MRC scheme is carried out in two phases.

- TAS Phase: Since Alice has only a single RF chain, it has to send pilot sequence one by one for the channel estimation at Bob. Furthermore, we assume that Bob can perfectly estimate the CSI corresponding to each transmit antenna. After that, Bob selects a transmit antenna associated with the best instantaneous SNR, and feeds back the optimal antenna index to Alice. The feedback information can be represented by a binary vector with $B=\left\lceil\log N_{t}\right\rceil$ bits.

- Transmission Phase: During the information transmission, the CSI corresponding to the optimal antenna should be estimated again for decoding at Bob. Using the pilot sequence designed in the information block, Bob can obtain the accurate CSI of the main channel. Then, Bob requires to feed back the instantaneous SNR of the main channel to Alice for wiretap code construction. ${ }^{1}$

Since the eavesdropper channel is not available, the TAS criterion used is to select the highest post-processing SNR for Bob, which is entirely determined by the CSI of the main channel. As such, the index of the selected antenna, $u^{*}$, is determined by

$$
u^{*}=\arg \max _{1 \leq u \leq N_{t}}\|\mathbf{H}(u)\|,
$$

where $\mathbf{H}(u)$ corresponds to the $u$ th column of $\mathbf{H}$.

Nevertheless, from Eve's point of view, the optimum TAS scheme for Bob will be a random TAS, as the main channel and the eavesdropper channel are uncorrelated. Consequently, the TAS scheme will provide the diversity gain to Bob but not to Eve; secure communications can thus be realized.

Alice encodes each message $\mathbf{w}$ into a codeword $\mathbf{x}=$ $[x(1), x(2), \cdots, x(n)]$, where $n$ is the length of $\mathbf{x}$, and the transmitted codeword is subject to an average power constraint $\frac{1}{n} \sum_{i=1}^{n} \mathbb{E}\left\{|x(i)|^{2}\right\} \leq P$. The received signals at Bob and Eve at time $i$ are, respectively, written as

$$
\begin{aligned}
& \mathbf{y}(i)=\mathbf{h} x(i)+\mathbf{n}_{b}, \\
& \mathbf{z}(i)=\mathbf{g} x(i)+\mathbf{n}_{e},
\end{aligned}
$$

where $\mathbf{h}$ denotes the $N_{r} \times 1$ vector for the main channel $\mathbf{H}\left(u^{*}\right)$, and $\mathbf{g}$ denotes the $N_{e} \times 1$ vector for the eavesdropper channel between the $u^{*}$ th transmit antenna at Alice and Eve, i.e., $\mathbf{g} \triangleq$ $\mathbf{G}\left(u^{*}\right)$. Also, $\mathbf{n}_{b}$ and $\mathbf{n}_{e}$ are additive white complex Gaussian noise at Bob and Eve, respectively. We assume that $\mathbf{n}_{b} \sim$ $\mathcal{C N}\left(\mathbf{0}, \sigma_{b}^{2} \mathbf{I}_{N_{r}}\right), \mathbf{n}_{e} \sim \mathcal{C N}\left(\mathbf{0}, \sigma_{e}^{2} \mathbf{I}_{N_{e}}\right)$.

We consider the MRC reception scheme to be employed at both Bob and Eve since MRC always outperforms other diversity combining schemes [26]. ${ }^{2}$ As a result, the instantaneous

\footnotetext{
${ }^{1}$ Different from the conventional TAS-MRC scheme without secrecy consideration, Bob has to feed back the received SNR value to Alice for wiretap code construction, in addition to the optimal transmit antenna index.

${ }^{2} \mathrm{SC}$ employed at the eavesdropper was also considered in [22]. In this case, the TAS-MRC scheme has a better secrecy performance than that of MRC scheme at the eavesdropper. However, in this paper, to highlight the impact of imperfect feedbacks on the secrecy performance, we assume that the eavesdropper has a higher computational capability, i.e., MRC scheme is adopted at the eavesdropper. The secrecy performance analysis for the SC reception scheme at the eavesdropper can be conducted in a similar manner.
}

SNRs of the main channel and the eavesdropper channel can be, respectively, given by

$$
\begin{aligned}
\gamma_{m} & =\frac{\|\mathbf{h}\|^{2} P}{\sigma_{b}^{2}}, \\
\gamma_{w} & =\frac{\|\mathbf{g}\|^{2} P}{\sigma_{e}^{2}} .
\end{aligned}
$$

The achievable secrecy rate $R_{s}$ can be expressed as [27]

$$
R_{s}=\left\{\begin{array}{cl}
R_{m}-R_{w}, & \text { if } \gamma_{m}>\gamma_{w}, \\
0, & \text { if } \gamma_{m} \leq \gamma_{w},
\end{array}\right.
$$

where $R_{m} \triangleq \log \left(1+\gamma_{m}\right)$ and $R_{w} \triangleq \log \left(1+\gamma_{w}\right)$ are the achievable instantaneous rates at Bob and Eve, respectively.

\section{B. Preliminaries}

The secrecy performance of TAS/MRC-based PLS scheme for MIMO wiretap channels with perfect feedbacks has been studied in the literature, e.g., [22]. With perfect feedbacks, it is well known that the probability density function (pdf) of the SNR for the main channel $\gamma_{m}$, denoted by $f\left(\gamma_{m}\right)$, follows the ordered $\chi^{2}$ distribution, given by [16]

$$
\begin{aligned}
f\left(\gamma_{m}\right) & =\frac{N_{t}}{\left(N_{r}-1\right) !} \\
\times \sum_{i=0}^{N_{t}-1}(-1)^{i} & \left(\begin{array}{c}
N_{t}-1 \\
i
\end{array}\right) \exp \left(-\frac{(i+1) \gamma_{m}}{\bar{\gamma}_{m}}\right) \\
& \times \sum_{j=0}^{i\left(N_{r}-1\right)} \xi_{j i}\left(\frac{1}{\bar{\gamma}_{m}}\right)^{j+N_{r}} \gamma_{m}^{j+N_{r}-1},
\end{aligned}
$$

where $\xi_{j i}=\sum_{x=a}^{b} \frac{\xi_{x(i-1)}}{(j-x) !}$, with $a=\max \left\{0, j-\left(N_{r}-1\right)\right\}$, $b=\min \left\{j,(i-1)\left(N_{r}-1\right)\right\}, \xi_{j 0}=\xi_{0 i}=1, \xi_{j 1}=\frac{1}{j !}$, and $\xi_{1 i}=i$, and $\bar{\gamma}_{m}=\frac{P}{\sigma_{b}^{2}}$ is the average SNR per receive antenna.

In addition, when TAS is not employed at the transmitter (i.e., $N_{t}=1$ ), the pdf and cumulative distribution function (cdf) of the SNR at the MRC combiner output, denoted $p(\gamma)$ and $P(\gamma)$, respectively, are reduced to [15]

$$
\left\{\begin{array}{l}
p(\gamma)=\frac{\gamma^{N_{r}-1}}{\left(N_{r}-1\right) !}\left(\frac{1}{\bar{\gamma}}\right)^{N_{r}} \exp \left(-\frac{\gamma}{\bar{\gamma}}\right), \\
P(\gamma)=\frac{\Gamma\left(N_{r}, \frac{\gamma}{\bar{\gamma}}\right)}{\left(N_{r}-1\right) !},
\end{array}\right.
$$

where $\Gamma(a, x)=\int_{0}^{x} t^{a-1} e^{-t} d t$ denotes the lower incomplete Gamma function.

Using (8), the pdf of $\gamma_{w}$, denoted by $f\left(\gamma_{w}\right)$, is [21]

$$
f\left(\gamma_{w}\right)=\frac{\gamma_{w}^{N_{e}-1}}{\left(N_{e}-1\right) !}\left(\frac{1}{\bar{\gamma}_{w}}\right)^{N_{e}} \exp \left(-\frac{\gamma_{w}}{\bar{\gamma}_{w}}\right),
$$

where $\bar{\gamma}_{w}=\frac{P}{\sigma_{e}^{2}}$ denotes the average SNR per receive antenna for the eavesdropper channel.

In this TAS-MRC scheme, due to the absence of the Eve's channel knowledge, Alice will transmit at an arbitrary rate $R_{0}$. For passive eavesdroppers, the wiretap code construction at Alice is based on the main channel capacity $R_{m}$ and $R_{0}$. The estimate of the eavesdropper's channel capacity is assumed as 
$\hat{R}_{w}=R_{m}-R_{0}$ [17], [24]. Therefore, if $R_{0}<R_{s}$, or the eavesdropper's channel is worse than Alice's estimate, i.e., $R_{w}<\hat{R}_{w}$, perfect secrecy can thus be obtained. Otherwise, if $R_{0}>R_{s}$, then $R_{w}>\hat{R}_{w}$ and such transmission may not be secure, leading to secrecy outage. Here, we consider the scenario in which the feedback of the optimal transmit antenna index is outdated and/or erroneous, and as such, the transmitter may not select the optimal antenna. In the subsequent sections, we will find new closed-form expressions for the probability of non-zero secrecy rate and secrecy outage probability when separately and jointly considering timedelayed feedback and erroneous feedback. Based on those exact expressions, asymptotic analysis is carried out, which completely characterizes the impacts of imperfect feedback on the secrecy performance.

\section{Performance with Time-Delayed Feedback}

In practical systems, TAS phase may exceed the coherent time of the channel. As a result, the main channel may have already changed the moment when Alice receives the feedback of the optimal antenna index because of the time-varying nature of the wireless channel. In this case, the optimal antenna is selected based on outdated CSI. Let $\mathbf{h}(n-\tau)$ denote the $\tau$ time-delayed main channel version of the current CSI $\mathbf{h}(n)$. We adopt the Gauss-Markov fading model to characterize the relationship between $\mathbf{h}(n-\tau)$ and $\mathbf{h}(n)$ [28], i.e.,

$$
\mathbf{h}(n)=\sqrt{\rho} \mathbf{h}(n-\tau)+\sqrt{1-\rho} \mathbf{e}(n),
$$

where $\mathbf{e}(n) \sim \mathcal{C N}\left(\mathbf{0}, \mathbf{I}_{N_{r}}\right)$ is the channel error vector, which is independent of $\mathbf{h}(n-\tau)$, and $\sqrt{\rho}$ is the correlation coefficient between $\mathbf{h}(n)$ and $\mathbf{h}(n-\tau)$, which is given by $\sqrt{\rho}=$ $J_{0}\left(2 \pi f_{d} \tau\right)$, where $J_{0}(\cdot)$ is the zeroth-order Bessel function of the first kind, and $f_{d}$ denotes the Doppler frequency.

Further, let $\gamma_{m}$ denote the time-delayed version of the current SNR $\tilde{\gamma}_{m}$, i.e., $\tilde{\gamma}_{m}=\|\mathbf{h}(n)\|^{2} P / \sigma_{b}^{2}$ and $\gamma_{m}=$ $\|\mathbf{h}(n-\tau)\|^{2} P / \sigma_{b}^{2}$. Note that the pdf of $\tilde{\gamma}_{m}$, denoted by $f\left(\tilde{\gamma}_{m}\right)$, can be expressed as [16], [25]

$$
f\left(\tilde{\gamma}_{m}\right)=\int_{0}^{\infty} p\left(\tilde{\gamma}_{m} \mid \gamma_{m}\right) f\left(\gamma_{m}\right) d \gamma_{m}
$$

where $p\left(\tilde{\gamma}_{m} \mid \gamma_{m}\right)$ denotes the pdf of $\tilde{\gamma}_{m}$ conditioned on $\gamma_{m}$, and $\tilde{\gamma}_{m}$ and $\gamma_{m}$ are two correlated $\chi^{2}$-distributed random variables. According to [29], the conditional pdf is given by

$$
\begin{aligned}
& p\left(\tilde{\gamma}_{m} \mid \gamma_{m}\right)=\frac{1}{(1-\rho)}\left(\frac{1}{\bar{\gamma}_{m}}\right)\left(\frac{\tilde{\gamma}_{m}}{\rho \gamma_{m}}\right)^{\frac{N_{r}-1}{2}} \\
& \quad \times \exp \left(-\frac{\rho \gamma_{m}+\tilde{\gamma}_{m}}{(1-\rho) \bar{\gamma}_{m}}\right) I_{N_{r}-1}\left(\frac{2 \sqrt{\rho \gamma_{m} \tilde{\gamma}_{m}}}{(1-\rho) \bar{\gamma}_{m}}\right),
\end{aligned}
$$

where $I_{v}(\cdot)$ is the modified Bessel function of the first kind with the order of $v$, and the correlation coefficient $\rho$ is given by $\rho=J_{0}^{2}\left(2 \pi f_{d} \tau\right)$.
Substituting (12) into (11), we can obtain the pdf of $\tilde{\gamma}_{m}$ as

$$
\begin{aligned}
& f\left(\tilde{\gamma}_{m}\right)=\frac{N_{t}}{\left(N_{r}-1\right) !} \\
& \times \sum_{i=0}^{N_{t}-1}(-1)^{i}\left(\begin{array}{c}
N_{t}-1 \\
i
\end{array}\right) \exp \left(-\frac{(i+1) \tilde{\gamma}_{m}}{(i(1-\rho)+1) \bar{\gamma}_{m}}\right) \\
& \quad \times \sum_{j=0}^{i\left(N_{r}-1\right)}\left\{\xi_{j i}\left(j+N_{r}-1\right) ! \sum_{k=0}^{j}\left(\begin{array}{l}
j \\
k
\end{array}\right)\left(\frac{1}{\bar{\gamma}_{m}}\right)^{k+N_{r}}\right. \\
& \left.\quad \times \frac{\rho^{k}(1-\rho)^{j-k}}{(i(1-\rho)+1)^{j+k+N_{r}}} \frac{\tilde{\gamma}_{m}^{k+N_{r}-1}}{\left(k+N_{r}-1\right) !}\right\} .
\end{aligned}
$$

When $\rho=1$, it corresponds to the case $\tau=0$, i.e., perfect feedback without delay and that (13) boils down to (7).

In addition, an important problem is the construction of wiretap code in the case of time-delayed feedback. To construct the corresponding wiretap code, Bob has to feed back the current SNR value $\tilde{\gamma}_{m}$ to Alice during transmission phase. We assume that the feedback of $\tilde{\gamma}_{m}$ is perfect. Thus, Alice will use the main channel capacity of $R_{m}=\log \left(1+\tilde{\gamma}_{m}\right)$ to construct the wiretap code and transmit the signal at the antenna selected based on $\mathbf{h}(n-\tau)$ [25]. Next, we will characterize the impact of the nonoptimal antenna selection caused by time-delayed feedback on the secrecy performance.

\section{A. The probability of non-zero secrecy rate}

According to (6), the probability of non-zero secrecy rate can be expressed as

$$
\begin{aligned}
\tilde{P}_{\text {non }} & =\operatorname{Pr}\left\{R_{s}>0\right\}=\operatorname{Pr}\left\{\tilde{\gamma}_{m}>\gamma_{w}\right\} \\
& =\int_{0}^{\infty} \int_{0}^{\int_{\gamma_{m}}} f\left(\gamma_{w}\right) f\left(\tilde{\gamma}_{m}\right) d \gamma_{w} d \tilde{\gamma}_{m} \\
& =\int_{0}^{\infty} \underbrace{\int_{0}^{\tilde{\gamma}_{m}} f\left(\gamma_{w}\right) d \gamma_{w}}_{P_{1}} f\left(\tilde{\gamma}_{m}\right) d \tilde{\gamma}_{m} .
\end{aligned}
$$

Using the following identity [30, (3.351.1)],

$$
\int_{0}^{u} x^{n} \exp (-\alpha x) d x=\frac{n !}{\alpha^{n+1}}-\exp (-\alpha u) \sum_{k=0}^{n} \frac{n !}{k !} \frac{u^{k}}{\alpha^{n-k+1}}
$$

the inner integral of (14) can be expressed as

$$
P_{1}=\int_{0}^{\tilde{\gamma}_{m}} f\left(\gamma_{w}\right) d \gamma_{w}=1-\exp \left(-\frac{\tilde{\gamma}_{m}}{\bar{\gamma}_{w}}\right) \sum_{k=0}^{N_{e}-1} \frac{1}{k !}\left(\frac{\tilde{\gamma}_{m}}{\bar{\gamma}_{w}}\right)^{k} .
$$

Now, substituting (16) into (14), and using the identity [30, (3.351.3)],

$$
\int_{0}^{\infty} x^{n} \exp (-\alpha x) d x=\frac{n !}{\alpha^{n+1}}
$$


we have

$$
\begin{aligned}
\tilde{P}_{\text {non }}= & \int_{0}^{\infty} P_{1} f\left(\tilde{\gamma}_{m}\right) d \tilde{\gamma}_{m} \\
= & 1-\frac{N_{t}}{\left(N_{r}-1\right) !} \sum_{k=0}^{N_{e}-1} \sum_{i=0}^{N_{t}-1}(-1)^{i}\left(\begin{array}{c}
N_{t}-1 \\
i
\end{array}\right) \\
& \times \sum_{j=0}^{i\left(N_{r}-1\right)}\left\{\xi_{j i}\left(j+N_{r}-1\right) ! \sum_{n=0}^{j}\left(\begin{array}{c}
k+n+N_{r}-1 \\
k
\end{array}\right)\right. \\
& \left.\times\left(\begin{array}{c}
j \\
n
\end{array}\right) \frac{\rho^{n}(1-\rho)^{j-n}}{(i(1-\rho)+1)^{j-k}} \frac{\bar{\gamma}_{m}^{k} \bar{\gamma}_{w}^{n+N_{r}}}{\mu^{k+n+N_{r}}}\right\},
\end{aligned}
$$

where $\mu=(i(1-\rho)+1) \bar{\gamma}_{m}+(i+1) \bar{\gamma}_{w}$.

Note that $\rho=1$ corresponds to the case of perfect feedback. In this case, the probability of non-zero secrecy rate in (18) reduces to

$$
\begin{aligned}
P_{\text {non }}= & 1-\frac{N_{t}}{\left(N_{r}-1\right) !} \sum_{k=0}^{N_{e}-1} \sum_{i=0}^{N_{t}-1}(-1)^{i}\left(\begin{array}{c}
N_{t}-1 \\
i
\end{array}\right) \\
& \sum_{j=0}^{i\left(N_{r}-1\right)}\left\{\xi_{j i} \frac{\left(k+j+N_{r}-1\right) !}{k !} \frac{\bar{\gamma}_{m}^{k} \bar{\gamma}_{w}^{j+N_{r}}}{\mu^{k+j+N_{r}}}\right\},
\end{aligned}
$$

where $\mu=\bar{\gamma}_{m}+(i+1) \bar{\gamma}_{w}$.

In addition, if $\rho=0$ in (18), then the current channel will be fully independent of the channel the moment Bob selects transmit antenna, and Alice will select an arbitrary antenna to transmit data. In this case, the probability of non-zero secrecy rate of the random TAS scheme can be expressed as

$$
\begin{aligned}
P_{\text {non }}= & 1-\frac{N_{t}}{\left(N_{r}-1\right) !} \sum_{k=0}^{N_{e}-1}\left(\begin{array}{c}
k+N_{r}-1 \\
k
\end{array}\right) \frac{\bar{\gamma}_{m}^{k} \bar{\gamma}_{w}^{N_{r}}}{\left(\bar{\gamma}_{m}+\bar{\gamma}_{w}\right)^{k+N_{r}}} \\
& \sum_{i=0}^{N_{t}-1}\left\{(-1)^{i}\left(\begin{array}{c}
N_{t}-1 \\
i
\end{array}\right) \sum_{j=0}^{i\left(N_{r}-1\right)} \xi_{j i} \frac{\left(j+N_{r}-1\right) !}{(i+1)^{j+N_{r}}}\right\} \\
= & 1-\sum_{k=0}^{N_{e}-1}\left(\begin{array}{c}
N_{r}+k-1 \\
k
\end{array}\right) \frac{\bar{\gamma}_{m}^{k} \bar{\gamma}_{w}^{N_{r}}}{\left(\bar{\gamma}_{m}+\bar{\gamma}_{w}\right)^{k+N_{r}}} .
\end{aligned}
$$

Remark 1: In the case of single transmit antenna, i.e., $N_{t}=$ 1 , the corresponding probability of non-zero secrecy rate can be obtained from (19) as

$$
P_{\text {non }}=1-\sum_{k=0}^{N_{e}-1}\left(\begin{array}{c}
N_{r}+k-1 \\
k
\end{array}\right) \frac{\bar{\gamma}_{m}^{k} \bar{\gamma}_{w}^{N_{r}}}{\left(\bar{\gamma}_{m}+\bar{\gamma}_{w}\right)^{k+N_{r}}} .
$$

This concurs with the result in [18, (3)], i.e., the MRC scheme with perfect feedbacks. Moreover, it can be observed that the probability of non-zero secrecy rate in (21) is equal to that of the random TAS in (20), which means that when $\rho=0$, the random TAS scheme cannot obtain any secrecy performance gain. In other words, when the feedback of the optimal antenna index is severely outdated (i.e., $\rho=0$ ), the gain from TAS vanishes.

Remark 2: In the case of single receive antenna, i.e., $N_{r}=$ 1 , the corresponding probability of non-zero secrecy rate can be obtained from (19) as

$$
P_{\text {non }}=1-\sum_{i=0}^{N_{t}}(-1)^{i}\left(\begin{array}{c}
N_{t} \\
i
\end{array}\right)\left(1+i \frac{\bar{\gamma}_{w}}{\bar{\gamma}_{m}}\right)^{-N_{e}},
$$

which corresponds to $[21,(5)]$, i.e., TAS with perfect feedbacks. Thus, our results embrace the known results. Further, with $N_{t}=N_{r}=N_{e}=1,(21)$ and (22) reduce to [27]

$$
P_{\text {non }}=\frac{\bar{\gamma}_{m}}{\left(\bar{\gamma}_{m}+\bar{\gamma}_{w}\right)}
$$

Remark 3: Now, we derive the asymptotic results for both the cases when (1) $\bar{\gamma}_{m} \gg \bar{\gamma}_{w}$ and (2) $\bar{\gamma}_{w} \gg \bar{\gamma}_{m}$. From (18), it follows that when $\bar{\gamma}_{m} \gg \bar{\gamma}_{w}$, then we have (24) (see top of next page), which is anticipated. Similarly, when $\bar{\gamma}_{w} \gg \bar{\gamma}_{m}$, we obtain (25) which is also expected (see top of next page).

From (24) and (25), though the feedback from Bob to Alice is outdated, the probability of non-zero secrecy rate will still approach to one as long as the average channel gain of the main channel is higher than that of the eavesdropper channel. However, if $\bar{\gamma}_{w} \gg \bar{\gamma}_{m}$, a positive probability of non-zero secrecy rate will be impossible even with perfect feedback.

\section{B. Secrecy outage probability}

For a given target secrecy rate $R_{0}>0$, the secrecy outage probability is formulated as [27]

$$
\begin{aligned}
& \tilde{\varepsilon}_{\text {out }}=\operatorname{Pr}\left\{R_{s}<R_{0}\right\} \\
& =\operatorname{Pr}\left\{R_{m} \leq R_{0}\right\}+\operatorname{Pr}\left\{R_{s}<R_{0}, R_{m}>R_{0}\right\} \\
& =1-\int_{0}^{\infty} \underbrace{\int_{2^{R_{0}} \gamma_{w}+\left(2^{\left.R_{0}-1\right)}\right.}^{\infty} f\left(\tilde{\gamma}_{m}\right) d \tilde{\gamma}_{m}}_{P_{2}} f\left(\gamma_{w}\right) d \gamma_{w} .
\end{aligned}
$$

The meaning of (26) is twofold. First, it includes the outage probability for the case where Alice does not transmit, i.e., $R_{m} \leq R_{0}$. It also gives the metric for the case where the message transmission is not perfectly secure, i.e., there exists some information leakage to Eve [17], [27].

With some tedious mathematical manipulations, we can calculate the inner integral $P_{2}$ as (27), where $\hat{\gamma}_{w}=2^{R_{0}} \gamma_{w}+$ $\left(2^{R_{0}}-1\right)$. Furthermore, replacing the value of $f\left(\gamma_{w}\right)$ and $P_{2}$, we obtain the closed-form expression of (26) as (28), where $\eta=\frac{(i+1)}{(i(1-\rho)+1) \bar{\gamma}_{m}}$.

Note that when $\rho=1$, it corresponds to the case of perfect feedback and the secrecy outage probability in (28) is reduced to (29) with $\eta=\frac{i+1}{\bar{\gamma}_{m}}$. On the other hand, in the case of $\rho=0$, the secrecy outage probability is given by (30).

Remark 4: It is also mentioned that the probability of nonzero secrecy rate $\tilde{P}_{\text {non }}$ can be evaluated via $\tilde{P}_{\text {non }}=1-$ $\tilde{\varepsilon}_{\text {out }}\left(R_{0}=0\right)$, and the same result can be obtained [23].

Remark 5: In the case of single transmit antenna, i.e., $N_{t}=$ 1 , the secrecy outage probability is derived using (29) as

$$
\begin{gathered}
\varepsilon_{\text {out }}=1-\frac{1}{\left(N_{e}-1\right) !} \sum_{n=0}^{N_{r}-1} \frac{1}{n !} \frac{1}{\bar{\gamma}_{m}^{n} \bar{\gamma}_{w}^{N_{e}}} \exp \left(-\frac{2^{R_{0}}-1}{\bar{\gamma}_{m}}\right) \\
\times\left[\sum_{v=0}^{n}\left(\begin{array}{l}
n \\
v
\end{array}\right)\left(2^{R_{0}}-1\right)^{n-v} \frac{2^{R_{0} v}\left(N_{e}+v-1\right) !}{\left(\frac{1}{\bar{\gamma}_{w}}+\frac{2^{R_{0}}}{\bar{\gamma}_{m}}\right)^{N_{e}+v}}\right],
\end{gathered}
$$




$$
\begin{aligned}
\tilde{P}_{\text {non }} \approx 1- & \frac{N_{t}}{\left(N_{r}-1\right) !} \sum_{k=0}^{N_{e}-1} \sum_{i=0}^{N_{t}-1}(-1)^{i}\left(\begin{array}{c}
N_{t}-1 \\
i
\end{array}\right) \\
& \times \sum_{j=0}^{i\left(N_{r}-1\right)}\left\{\xi_{j i}\left(j+N_{r}-1\right) ! \sum_{n=0}^{j}\left(\begin{array}{c}
k+n+N_{r}-1 \\
k
\end{array}\right) \times\left(\begin{array}{l}
j \\
n
\end{array}\right) \frac{\rho^{n}(1-\rho)^{j-n}}{(i(1-\rho)+1)^{j+n+N_{r}}}\left(\frac{\bar{\gamma}_{w}}{\bar{\gamma}_{m}}\right)^{n+N_{r}}\right\}=1
\end{aligned}
$$

$$
\begin{aligned}
\tilde{P}_{\text {non }} & \approx 1-\frac{N_{t}}{\left(N_{r}-1\right) !} \sum_{k=0}^{N_{e}-1} \sum_{i=0}^{N_{t}-1}(-1)^{i}\left(\begin{array}{c}
N_{t}-1 \\
i
\end{array}\right) \\
& \times \sum_{j=0}^{i\left(N_{r}-1\right)}\left\{\xi_{j i}\left(j+N_{r}-1\right) ! \sum_{n=0}^{j}\left(\begin{array}{c}
k+n+N_{r}-1 \\
k
\end{array}\right) \times\left(\begin{array}{l}
j \\
n
\end{array}\right) \frac{\rho^{n}(1-\rho)^{j-n}}{(i(1-\rho)+1)^{j-k}} \frac{1}{(i+1)^{k+n+N_{r}}}\left(\frac{\bar{\gamma}_{m}}{\bar{\gamma}_{w}}\right)^{k}\right\} \\
& =1-\frac{N_{t}}{\left(N_{r}-1\right) !} \sum_{i=0}^{N_{t}-1}(-1)^{i}\left(\begin{array}{c}
N_{t}-1 \\
i
\end{array}\right) \sum_{j=0}^{i\left(N_{r}-1\right)}\left\{\xi_{j i} \times \frac{\left(j+N_{r}-1\right) !}{(i(1-\rho)+1)^{j}} \sum_{n=0}^{j}\left(\begin{array}{l}
j \\
n
\end{array}\right) \frac{\rho^{n}(1-\rho)^{j-n}}{(i+1)^{n+N_{r}}}\right\}=0
\end{aligned}
$$

$$
\begin{aligned}
P_{2} & =\int_{\hat{\gamma}_{w}}^{\infty} f\left(\tilde{\gamma}_{m}\right) d \tilde{\gamma}_{m} \\
& =\frac{N_{t}}{\left(N_{r}-1\right) !} \sum_{i=0}^{N_{t}-1}(-1)^{i}\left(\begin{array}{c}
N_{t}-1 \\
i
\end{array}\right) \times \sum_{j=0}^{i\left(N_{r}-1\right)}\left\{\xi_{j i}\left(j+N_{r}-1\right) ! \sum_{k=0}^{j}\left(\begin{array}{l}
j \\
k
\end{array}\right)\right. \\
& \left.\times\left(\frac{1}{\bar{\gamma}_{m}}\right)^{k+N_{r}} \frac{\rho^{k}(1-\rho)^{j-k}}{(i(1-\rho)+1)^{j+k+N_{r}}} \frac{1}{\left(k+N_{r}-1\right) !} \times \int_{\hat{\gamma}_{w}}^{\infty} \tilde{\gamma}_{m}^{k+N_{r}-1} \exp \left(-\frac{(i+1) \tilde{\gamma}_{m}}{(i(1-\rho)+1) \bar{\gamma}_{m}}\right) d \tilde{\gamma}_{m}\right\} \\
& =\frac{N_{t}}{\left(N_{r}-1\right) !} \sum_{i=0}^{N_{t}-1}(-1)^{i}\left(\begin{array}{c}
N_{t}-1 \\
i
\end{array}\right) \sum_{j=0}^{i\left(N_{r}-1\right)}\left\{\xi_{j i}\left(j+N_{r}-1\right) ! \sum_{k=0}^{j}\left(\begin{array}{l}
j \\
k
\end{array}\right)\left(\frac{1}{\bar{\gamma}_{m}}\right)^{k+N_{r}} \exp \left(-\eta \hat{\gamma}_{w}\right)\right. \\
& \left.\times \frac{\rho^{k}(1-\rho)^{j-k}}{(i(1-\rho)+1)^{j+k+N_{r}}} \sum_{n=0}^{k+N_{r}-1} \frac{1}{n !} \frac{\hat{\gamma}_{w}^{n}}{\eta^{k+N_{r}-n}}\right\},
\end{aligned}
$$

$$
\begin{aligned}
\tilde{\varepsilon}_{\text {out }}=1-\frac{N_{t}}{\left(N_{r}-1\right) !\left(N_{e}-1\right) !} \sum_{i=0}^{N_{t}-1}(-1)^{i}\left(\begin{array}{c}
N_{t}-1 \\
i
\end{array}\right) \times \sum_{j=0}^{i\left(N_{r}-1\right)}\left\{\xi_{j i}\left(j+N_{r}-1\right) ! \sum_{k=0}^{j}\left(\begin{array}{l}
j \\
k
\end{array}\right) \sum_{n=0}^{k+N_{r}-1} \frac{1}{n !}\right. \\
\left.\times \frac{\rho^{k}(1-\rho)^{j-k}}{(i(1-\rho)+1)^{j+n}} \frac{\exp \left(-\eta\left(2^{R_{0}}-1\right)\right)}{(i+1)^{k+N_{r}-n}} \frac{1}{\bar{\gamma}_{m}^{n} \bar{\gamma}_{w}^{N_{e}}} \times\left[\sum_{v=0}^{n}\left(\begin{array}{l}
n \\
v
\end{array}\right)\left(2^{R_{0}}-1\right)^{n-v} \frac{2^{R_{0} v}\left(N_{e}+v-1\right) !}{\left(\frac{1}{\bar{\gamma}_{w}}+2^{R_{0}} \eta\right)^{N_{e}+v}}\right]\right\},
\end{aligned}
$$

$$
\begin{aligned}
\varepsilon_{\text {out }}=1-\frac{N_{t}}{\left(N_{r}-1\right) !\left(N_{e}-1\right) !} \sum_{i=0}^{N_{t}-1}(-1)^{i} \times\left(\begin{array}{c}
N_{t}-1 \\
i
\end{array}\right) \sum_{j=0}^{i\left(N_{r}-1\right)}\left\{\xi_{j i}\left(j+N_{r}-1\right) !\right. \\
\left.\quad \times \sum_{n=0}^{j+N_{r}-1} \frac{1}{n !} \frac{\exp \left(-\eta\left(2^{R_{0}}-1\right)\right)}{(i+1)^{j+N_{r}-n} \bar{\gamma}_{m}^{n} \bar{\gamma}_{w}^{N_{e}}}\left[\sum_{v=0}^{n}\left(\begin{array}{c}
n \\
v
\end{array}\right) \times\left(2^{R_{0}}-1\right)^{n-v} 2^{R_{0} v} \frac{\left(N_{e}+v-1\right) !}{\left(\frac{1}{\bar{\gamma}_{w}}+2^{R_{0}} \eta\right)^{N_{e}+v}}\right]\right\},
\end{aligned}
$$




$$
\begin{aligned}
\varepsilon_{\text {out }} & =1-\frac{N_{t}}{\left(N_{r}-1\right) !\left(N_{e}-1\right) !} \sum_{i=0}^{N_{t}-1}(-1)^{i}\left(\begin{array}{c}
N_{t}-1 \\
i
\end{array}\right) \\
& \times \sum_{j=0}^{i\left(N_{r}-1\right)}\left\{\xi_{j i} \frac{\left(j+N_{r}-1\right) !}{(i+1)^{j+N_{r}}} \sum_{n=0}^{N_{r}-1} \frac{1}{n !} \frac{\exp \left(-\frac{2^{R_{0}}-1}{\bar{\gamma}_{m}}\right)}{\bar{\gamma}_{m}^{n} \bar{\gamma}_{w}^{N_{e}}}\left[\sum_{v=0}^{n}\left(\begin{array}{c}
n \\
v
\end{array}\right)\left(2^{R_{0}}-1\right)^{n-v} \frac{2^{R_{0} v}\left(N_{e}+v-1\right) !}{\left(\frac{1}{\bar{\gamma}_{w}}+\frac{2^{R_{0}}}{\bar{\gamma}_{m}}\right)^{N_{e}+v}}\right]\right\} \\
& =1-\frac{1}{\left(N_{e}-1\right) !} \sum_{n=0}^{N_{r}-1} \frac{1}{n !} \frac{1}{\bar{\gamma}_{m}^{n} \bar{\gamma}_{w}^{N_{e}}} \exp \left(-\frac{2^{R_{0}}-1}{\bar{\gamma}_{m}}\right)\left[\sum_{v=0}^{n}\left(\begin{array}{c}
n \\
v
\end{array}\right)\left(2^{R_{0}}-1\right)^{n-v} \frac{2^{R_{0} v}\left(N_{e}+v-1\right) !}{\left(\frac{1}{\bar{\gamma}_{w}}+\frac{2^{R_{0}}}{\bar{\gamma}_{m}}\right)^{N_{e}+v}}\right]
\end{aligned}
$$

which corresponds to $[18,(6)]$, i.e., MRC with perfect feedbacks. It can also be found that the secrecy outage probability of the random TAS scheme is the same as that of $N_{t}=1$. It infers that when the feedbacks are significantly outdated (i.e., $\rho=0$ ), the diversity gain from the TAS disappears, and only the MRC diversity at Bob remains.

Remark 6: In the case of single receive antenna, i.e., $N_{r}=$ 1 , the secrecy outage probability can be derived from (29) as

$$
\varepsilon_{\text {out }}=\sum_{i=0}^{N_{t}}(-1)^{i}\left(\begin{array}{c}
N_{t} \\
i
\end{array}\right) \exp \left(-i \frac{2^{R_{0}}-1}{\bar{\gamma}_{m}}\right)\left(i \frac{2^{R_{0}} \bar{\gamma}_{w}}{\bar{\gamma}_{m}}+1\right)^{-N_{e}}
$$

which aligns with [21, (9)]. For the special case of single antenna at all nodes, i.e., $N_{t}=N_{r}=N_{e}=1$, (31) and (32) reduce to $\varepsilon_{\text {out }}=1-\frac{\bar{\gamma}_{m}}{\bar{\gamma}_{m}+2^{R_{0}} \bar{\gamma}_{w}} \exp \left(-\frac{2^{R_{0}}-1}{\bar{\gamma}_{m}}\right)$ [21], [27].

\section{Asymptotic outage probability}

In the high SNR regime with $\bar{\gamma}_{m} \rightarrow \infty$, the asymptotic secrecy outage probability $\tilde{\varepsilon}_{\text {out }}^{\infty}$ can be expressed as

$$
\tilde{\varepsilon}_{\text {out }}^{\infty}=\Psi \bar{\gamma}_{m}^{-d}+o\left(\bar{\gamma}_{m}^{-d}\right),
$$

where $o(\cdot)$ denotes higher order terms. The asymptotic secrecy outage probability gives valuable insights via the secrecy diversity order $d$, which determines the slope of the outage probability curve, and the secrecy array gain $\Psi$, which characterizes the SNR gain relative to the reference curve $\bar{\gamma}_{m}^{-d}$ [22]. Next, we derive asymptotic expressions for the cases $\rho=1$ and $\rho \neq 1$ to reveal the diversity loss due to outdated feedback and how the secrecy outage probability behaves at high SNR.

- $\rho \neq 1$ : We first expand the exponential function in (13) using the Taylor series expansion given by $e^{-x}=$ $\sum_{k=0}^{\infty}(-x)^{k} / k$ !. Then, we only keep the first two terms and obtain the cdf of $\tilde{\gamma}_{m}$ as

$$
\begin{array}{r}
F\left(\tilde{\gamma}_{m}\right)=\frac{N_{t}}{\left(N_{r}-1\right) !} \sum_{i=0}^{N_{t}-1}(-1)^{i}\left(\begin{array}{c}
N_{t}-1 \\
i
\end{array}\right) \\
\sum_{j=0}^{i\left(N_{r}-1\right)} \xi_{j i} \frac{\left(j+N_{r}-1\right) !}{N_{r} !} \frac{(1-\rho)^{j}}{(i(1-\rho)+1)^{j+N_{r}}} \\
\left(\frac{\tilde{\gamma}_{m}}{\bar{\gamma}_{m}}\right)^{N_{r}}+o\left(\left(\frac{\tilde{\gamma}_{m}}{\bar{\gamma}_{m}}\right)^{N_{r}}\right) .
\end{array}
$$

By plugging (34) into (26), the asymptotic expression for the secrecy outage probability can be rewritten as

$$
\tilde{\varepsilon}_{\text {out }}^{\infty}=\Psi_{1} \bar{\gamma}_{m}^{-N_{r}}+o\left(\bar{\gamma}_{m}^{-N_{r}}\right)
$$

where

$$
\begin{gathered}
\Psi_{1}=\frac{N_{t}}{N_{r} !\left(N_{r}-1\right) !\left(N_{e}-1\right) !} \sum_{i=0}^{N_{t}-1}(-1)^{i}\left(\begin{array}{c}
N_{t}-1 \\
i
\end{array}\right) \\
\sum_{j=0}^{i\left(N_{r}-1\right)}\left\{\xi_{j i}\left(j+N_{r}-1\right) ! \frac{(1-\rho)^{j}}{(i(1-\rho)+1)^{j+N_{r}}}\right. \\
\left.\left[\sum_{v=0}^{N_{r}}\left(\begin{array}{c}
N_{r} \\
v
\end{array}\right) \frac{\left(2^{R_{0}}-1\right)^{N_{r}-v}}{2^{-R_{0} v} \bar{\gamma}_{w}^{-v}}\left(N_{e}+v-1\right) !\right]\right\} .
\end{gathered}
$$

- $\rho=1$ : Similarly, we obtain the first non-zero order expansion of the cdf of $\gamma_{m}$ from (7) as

$$
F\left(\gamma_{m}\right)=\frac{1}{\left(N_{r} !\right)^{N_{t}}}\left(\frac{\gamma_{m}}{\bar{\gamma}_{m}}\right)^{N_{t} N_{r}}+o\left(\left(\frac{\gamma_{m}}{\bar{\gamma}_{m}}\right)^{N_{t} N_{r}}\right) .
$$

Thus, in this case, the asymptotic secrecy outage probability can be obtained as

$$
\tilde{\varepsilon}_{\text {out }}^{\infty}=\Psi_{2} \bar{\gamma}_{m}^{-N_{t} N_{r}}+o\left(\bar{\gamma}_{m}^{-N_{t} N_{r}}\right)
$$

where

$$
\begin{aligned}
& \Psi_{2}=\frac{1}{\left(N_{r} !\right)^{N_{t}}\left(N_{e}-1\right) !} \\
& \quad\left[\sum_{i=0}^{N_{t} N_{r}}\left(\begin{array}{c}
N_{t} N_{r} \\
i
\end{array}\right) \frac{\left(2^{R_{0}}-1\right)^{N_{t} N_{r}-i}}{2^{-R_{0} i} \bar{\gamma}_{w}^{-i}}\left(N_{e}+i-1\right) !\right] .
\end{aligned}
$$

From above, we can observe that with perfect feedbacks, the TAS-MRC scheme can obtain the secrecy diversity order of $N_{t} N_{r}$. However, when the feedbacks are outdated, the desired diversity order cannot be realized and only the MRC diversity gain remains. The secrecy diversity order is independent of $N_{e}$, which only affects the secrecy array gain $\Psi$. In addition, using the inequality $\frac{\left(N_{e}+i-1\right) !}{\left(N_{e}-1\right) !} \geq N_{e}^{i}$, it can also be inferred that the secrecy array gain $\Psi_{1}$ and $\Psi_{2}$ are the monotonously increasing functions of $N_{e}$. Moreover, we assume that the total number of transmit antenna and receive antenna is fixed, i.e., $N_{t}+N_{r}=$ $N_{\text {total }}$. Thus, an intuitive idea is to allocate the best number of antennas to Alice and Bob for minimizing the secrecy outage probability. When $\rho \neq 1$, we should allocate more antennas to 
Bob, i.e., $N_{t}=1, N_{r}=N_{\text {total }}-1$. However, when $\rho=1$, a lower bound on the asymptotic secrecy outage probability $\tilde{\varepsilon}_{\text {out }}^{\infty}$ can be given by $\tilde{\varepsilon}_{\text {out }}^{\infty} \geq \frac{1}{\left(N_{r} !\right)^{N_{t}}}\left(\frac{2^{R_{0}}-1+2^{R_{0}} N_{e} \bar{\gamma}_{w}}{\bar{\gamma}_{m}}\right)^{N_{t} N_{r}}$. We observe that the first term of the lower bound $\frac{1}{\left(N_{r} !\right)^{N_{t}}}$ stands in a dominant position at high SNR. Hence, we can minimize the function $1 /\left[\left((1-\alpha) N_{\text {total }}\right) !\right]^{\alpha N_{\text {total }}}$ to approximately determine the best asymptotic allocation, where $\alpha=N_{t} / N_{\text {total }}$. Unfortunately, it is a non-linear function of $\alpha$ and the best value is dependent on $N_{\text {total }}$. We will carry out numerical simulations to characterize this analysis in Section VI.

\section{Performance with Erroneous Feedback}

In this section, we consider the case that the feedback of the optimal antenna index is not outdated, but may be received in error due to an unreliable feedback channel. In other words, the coherent time of the main channel is sufficiently great for TAS phase. The construction of wiretap code at Alice is similar to Section III. We denote $P_{e}$ as the probability of a single-bit feedback error. Without any loss of generality, the number of transmit antennas is assumed to be equal to the power of two (i.e., $N_{t}=2^{B}$ ), the feedback bit-errors are independent, and then the probability of feedback error, i.e., Alice erroneously selects any non-optimal transmit antenna for transmission, is determined by $\varepsilon=1-\left(1-P_{e}\right)^{B}$. Thus, with probability of $1-\varepsilon$, Alice will select correctly the optimal transmit antenna. However, with probability of $\varepsilon$, the feedback from Bob to Alice will be incorrect and Alice will select a transmit antenna with its SNR not being the maximum at Bob. In the erroneous feedback case any non-maximum SNRs will be used with the equal probability $1 /\left(N_{t}-1\right)$. Using the order statistics [31], the pdf of a random sample within those $\left(N_{t}-1\right)$ SNRs, denoted by $f_{c}\left(\gamma_{m}\right)$, can be derived as

$$
\begin{aligned}
f_{c}\left(\gamma_{m}\right)= & \frac{N_{t}}{N_{t}-1} \sum_{l=1}^{N_{t}-1}\left(\begin{array}{c}
N_{t}-1 \\
l-1
\end{array}\right) p\left(\gamma_{m}\right) \\
& \times\left[P\left(\gamma_{m}\right)\right]^{l-1}\left[1-P\left(\gamma_{m}\right)\right]^{N_{t}-l} \\
= & \frac{N_{t} p\left(\gamma_{m}\right)-f\left(\gamma_{m}\right)}{N_{t}-1},
\end{aligned}
$$

where $p\left(\gamma_{m}\right)$ and $P\left(\gamma_{m}\right)$ are given by (8), and $f\left(\gamma_{m}\right)$ is given by (7). As a result, the pdf of the SNR for the main channel with erroneous feedback, denoted by $f^{(e)}\left(\gamma_{m}\right)$, is given as

$$
\begin{aligned}
f^{(e)}\left(\gamma_{m}\right) & =(1-\varepsilon) f\left(\gamma_{m}\right)+\varepsilon f_{c}\left(\gamma_{m}\right) \\
& =(1-\chi) f\left(\gamma_{m}\right)+\chi p\left(\gamma_{m}\right),
\end{aligned}
$$

where $\chi \triangleq{\frac{N_{t} \varepsilon}{\left(N_{t}-1\right)}}^{3}$.

\section{A. The probability of non-zero secrecy rate}

It can be seen from (41) that $f^{(e)}\left(\gamma_{m}\right)$, the pdf of the SNR with erroneous feedback, is a linear combination of $f\left(\gamma_{m}\right)$, the pdf of the TAS/MRC-based SNR with perfect feedback, and

\footnotetext{
${ }^{3}$ When $N_{t}=1$, we denote $\chi=0$.
}

$p\left(\gamma_{m}\right)$, the pdf of the MRC-based SNR. Thus, the probability of non-zero secrecy rate with erroneous feedback is found as

$$
\begin{aligned}
P_{\text {non }}^{(e)} & =\operatorname{Pr}\left\{R_{s}>0\right\} \\
& =\int_{0}^{\infty} \int_{0}^{\gamma_{m}} f\left(\gamma_{w}\right) f^{(e)}\left(\gamma_{m}\right) d \gamma_{w} d \gamma_{m} \\
& =(1-\chi) \int_{0}^{\infty} \int_{0}^{\gamma_{m}} f\left(\gamma_{w}\right) f\left(\gamma_{m}\right) d \gamma_{w} d \gamma_{m} \\
& +\chi \int_{0}^{\infty} \int_{0}^{\gamma_{m}} f\left(\gamma_{w}\right) p\left(\gamma_{m}\right) d \gamma_{w} d \gamma_{m} \\
& =(1-\chi) P_{\text {non }}\left(N_{t}\right)+\chi P_{\text {non }}(1),
\end{aligned}
$$

where $P_{\text {non }}\left(N_{t}\right)$ is given by (19), and $P_{\text {non }}(1)$ is the special case of $P_{\text {non }}\left(N_{t}\right)$ with $N_{t}=1$, given by $(21)$.

Remark 7: From (42), it can be found that the probability of non-zero secrecy rate is a decreasing function of $\chi$ since $P_{\text {non }}\left(N_{t}\right)$ is always not smaller than $P_{\text {non }}(1)$. Furthermore, according to $\chi \triangleq \frac{N_{t} \varepsilon}{\left(N_{t}-1\right)}$, it is concluded that the probability of non-zero secrecy rate decreases with $P_{e}$, or erroneous feedback degrades the secrecy performance.

\section{B. Secrecy outage probability}

Similar to the analysis in Section IV-A, the secrecy outage probability with erroneous feedback can also be expressed as a linear combination of the secrecy outage probability for the TAS-MRC scheme with perfect feedback and the secrecy outage probability for the MRC scheme, i.e., (43), where $\varepsilon_{\text {out }}\left(N_{t}\right)$ is given by (29), and $\varepsilon_{\text {out }}(1)$ is the special case of $\varepsilon_{\text {out }}\left(N_{t}\right)$ with $N_{t}=1$, which is obtained in (31).

Remark 8: Note that the secrecy outage probability in (43) is an increasing function of $\chi$ because $\varepsilon_{\text {out }}(1) \geq \varepsilon_{\text {out }}\left(N_{t}\right)$ always holds, which means that as $P_{e}$ increases, the secrecy outage probability will increase. Furthermore, as $P_{e} \rightarrow 1, \chi$ will approach one and $\varepsilon_{\text {out }}^{(e)}$ approaches $\varepsilon_{\text {out }}(1)$. It indicates that the severe feedback errors will cause a secrecy diversity gain loss and only MRC diversity at Bob can be guaranteed. Also, as $P_{e} \rightarrow 0$ and $\chi \rightarrow 0$, the performance gain from TAS-MRC can be obtained, as expected.

\section{Asymptotic outage probability}

Now, we proceed to evaluate the asymptotic expressions for the secrecy outage probability for the cases $P_{e}=0$ and $P_{e} \neq 0$, respectively.

- $P_{e}=0$ : From (43), $\varepsilon_{\text {out }}^{(e)}=\varepsilon_{\text {out }}\left(N_{t}\right)$, and hence,

$$
\varepsilon_{\text {out }}^{(e) \infty}=\Psi_{2} \bar{\gamma}_{m}^{-N_{t} N_{r}}+o\left(\bar{\gamma}_{m}^{-N_{t} N_{r}}\right),
$$

where the secrecy array gain $\Psi_{2}$ is given in (39).

- $P_{e} \neq 0$ : Since the first term in (43) can introduce the secrecy diversity order of $N_{t} N_{r}$ as shown in the case of $P_{e}=0$, the secrecy diversity order is expected to be between $N_{t} N_{r}$ and $N_{r}$ in the low to middle SNR regime. However, as $\bar{\gamma}_{m} \rightarrow \infty$, the second term will be more dominating at high SNRs. Thus, the asymptotic secrecy outage probability can be written as

$$
\varepsilon_{\text {out }}^{(e) \infty}=\Psi_{3} \bar{\gamma}_{m}^{-N_{r}}+o\left(\bar{\gamma}_{m}^{-N_{r}}\right),
$$




$$
\begin{aligned}
\varepsilon_{\text {out }}^{(e)} & =1-\int_{0}^{\infty} \int_{2^{R_{0}} \gamma_{w}+\left(2^{\left.R_{0}-1\right)}\right.}^{\infty} f^{(e)}\left(\gamma_{m}\right) f\left(\gamma_{w}\right) d \gamma_{m} d \gamma_{w} \\
& =(1-\chi)\left[1-\int_{0}^{\infty} \int_{2^{R_{0}} \gamma_{w}+\left(2^{\left.R_{0}-1\right)}\right.}^{\infty} f\left(\gamma_{m}\right) f\left(\gamma_{w}\right) d \gamma_{m} d \gamma_{w}\right]+\chi\left[1-\int_{0}^{\infty} \int_{2^{R_{0}} \gamma_{w}+\left(2^{\left.R_{0}-1\right)}\right.}^{\infty} p\left(\gamma_{m}\right) f\left(\gamma_{w}\right) d \gamma_{m} d \gamma_{w}\right] \\
& =(1-\chi) \varepsilon_{\text {out }}\left(N_{t}\right)+\chi \varepsilon_{\text {out }}(1)
\end{aligned}
$$

where

$$
\begin{aligned}
\Psi_{3}= & \frac{\chi}{N_{r} !\left(N_{e}-1\right) !} \\
& {\left[\sum_{i=0}^{N_{r}}\left(\begin{array}{c}
N_{r} \\
i
\end{array}\right) \frac{\left(2^{R_{0}}-1\right)^{N_{r}-i}}{2^{-R_{0} i} \bar{\gamma}_{w}^{-i}}\left(N_{e}+i-1\right) !\right] . }
\end{aligned}
$$

In addition, in order to more clearly characterize the change of the diversity order from low to middle SNRs, we can express the asymptotic secrecy outage probability as

$$
\varepsilon_{\text {out }}^{(e) \infty}=\Psi_{4} \bar{\gamma}_{m}^{-N_{r}}+o\left(\bar{\gamma}_{m}^{-N_{r}}\right),
$$

where $\Psi_{4}=\Psi_{3}+(1-\chi) \Psi_{2} \bar{\gamma}_{m}^{-N_{t}}$.

Notably, we can conclude that the expected diversity order of $N_{t} N_{r}$ cannot be realized with erroneous feedbacks, and only the diversity order of $N_{r}$ remains. In addition, it is mentioned that similar to Section III-C, the asymptotic allocation of a fixed number of antennas $N_{\text {total }}$ between Alice and Bob can also be established for $P_{e}=0$ and $P_{e} \neq 0$, respectively.

\section{Performance with Both Time-Delayed And ERRONEOUS FEEDBACKS}

In this section, we analyze the secrecy performance of the TAS-MRC scheme when both feedback delays and errors are present. With probability of $1-\varepsilon$, the feedback of the optimal antenna index is decoded correctly. However, the feedback is also delayed by $\tau$, and therefore Alice selects the transmit antenna corresponding to the delayed version of the maximum SNR. On the other hand, with probability of $\varepsilon$, the feedback Alice receives is decoded erroneously. Using the induced order statistics [31], the pdf of the delayed version of the nonmaximum SNR, denoted by $f_{c}\left(\tilde{\gamma}_{m}\right)$, can be expressed as [16]

$$
\begin{aligned}
f_{c}\left(\tilde{\gamma}_{m}\right) & =\int_{0}^{\infty} p\left(\tilde{\gamma}_{m} \mid \gamma_{m}\right) f_{c}\left(\gamma_{m}\right) d \gamma_{m} \\
& =\frac{N_{t} p\left(\tilde{\gamma}_{m}\right)-f\left(\tilde{\gamma}_{m}\right)}{N_{t}-1}
\end{aligned}
$$

where $p\left(\tilde{\gamma}_{m}\right)$ corresponds to the special case of $f\left(\tilde{\gamma}_{m}\right)$ with $N_{t}=1$, and $f_{c}\left(\gamma_{m}\right)$ is given by (40).

As such, the pdf of the SNR for the main channel with consideration of feedback errors and delays, denoted by $f^{(e)}\left(\tilde{\gamma}_{m}\right)$, is given as

$$
\begin{aligned}
f^{(e)}\left(\tilde{\gamma}_{m}\right) & =(1-\varepsilon) f\left(\tilde{\gamma}_{m}\right)+\varepsilon f_{c}\left(\tilde{\gamma}_{m}\right) \\
& =(1-\chi) f\left(\tilde{\gamma}_{m}\right)+\chi p\left(\tilde{\gamma}_{m}\right),
\end{aligned}
$$

where $\chi$ is defined in (41). We can observe that $p\left(\tilde{\gamma}_{m}\right)=$ $p\left(\gamma_{m}\right)$ and $p\left(\gamma_{m}\right)$ is given by (8), which is expected since if no TAS is employed, i.e., $N_{t}=1$, the feedback delay will not affect the pdf of the SNR for the TAS-based scheme.

\section{A. The probability of non-zero secrecy rate}

From (49), we also observe that $f^{(e)}\left(\tilde{\gamma}_{m}\right)$, the pdf of the SNR with both time-delayed and erroneous feedbacks, can be expressed as a linear combination of $f\left(\tilde{\gamma}_{m}\right)$, the pdf of the SNR for the TAS/MRC-based scheme with time-delayed feedbacks, and $p\left(\gamma_{m}\right)$, the pdf of the MRC-based SNR. Similar to the analysis presented in Section IV-A, the probability of non-zero secrecy rate with outdated and erroneous feedbacks can be expressed as a linear combination of the probability of non-zero secrecy rate for the TAS/MRC-based scheme with outdated feedbacks and the probability of non-zero secrecy rate for the MRC-based scheme. That is,

$$
\begin{aligned}
\tilde{P}_{\text {non }}^{(e)}= & \operatorname{Pr}\left\{R_{s}>0\right\} \\
= & \int_{0}^{\infty} \int_{0}^{\tilde{\gamma}_{m}} f\left(\gamma_{w}\right) f^{(e)}\left(\tilde{\gamma}_{m}\right) d \gamma_{w} d \tilde{\gamma}_{m} \\
= & (1-\chi) \int_{0}^{\infty} \int_{0}^{\tilde{\gamma}_{m}} f\left(\gamma_{w}\right) f\left(\tilde{\gamma}_{m}\right) d \gamma_{w} d \tilde{\gamma}_{m} \\
& +\chi \int_{0}^{\infty} \int_{0}^{\tilde{\gamma}_{m}} f\left(\gamma_{w}\right) p\left(\tilde{\gamma}_{m}\right) d \gamma_{w} d \tilde{\gamma}_{m} \\
= & (1-\chi) \tilde{P}_{\text {non }}\left(N_{t}\right)+\chi \tilde{P}_{\text {non }}(1),
\end{aligned}
$$

where $\tilde{P}_{\text {non }}\left(N_{t}\right)$ is given by (18). Likewise, $\tilde{P}_{\text {non }}(1)$ is the special case of $\tilde{P}_{\text {non }}\left(N_{t}\right)$ with $N_{t}=1$ and given by (21).

Remark 9: As in Remark 1, when $\rho=0$, the probability of non-zero secrecy rate for the TAS/MRC-based scheme with time-delayed feedbacks is the same as that of $N_{t}=1$, i.e., $\tilde{P}_{\text {non }}\left(N_{t}\right)=\tilde{P}_{\text {non }}(1)=P_{\text {non }}(1)$. Thus, $(50)$ reduces to $\tilde{P}_{\text {non }}^{(e)}=$ $P_{\text {non }}(1)$, meaning that when $\rho=0$, the probability of non-zero secrecy rate will be independent of $P_{e}$.

\section{B. Secrecy outage probability}

The secrecy outage probability with both outdated and erroneous feedbacks can also be expressed as a linear combination of the secrecy outage probability for the TAS/MRC-based scheme with outdated feedbacks and the secrecy outage probability for the MRC-based scheme, i.e., (51), where $\tilde{\varepsilon}_{\text {out }}\left(N_{t}\right)$ is given by (28) and $\tilde{\varepsilon}_{\text {out }}(1)$ is the special case of $\tilde{\varepsilon}_{\text {out }}\left(N_{t}\right)$ with $N_{t}=1$ and $\tilde{\varepsilon}_{\text {out }}(1)=\varepsilon_{\text {out }}(1)$.

Remark 10: As in Remark 5, in the case of $\rho=0$, the secrecy outage probability for the TAS/MRC-based scheme with time-delayed feedbacks is the same as that of $N_{t}=1$, i.e., $\tilde{\varepsilon}_{\text {out }}\left(N_{t}\right)=\tilde{\varepsilon}_{\text {out }}(1)=\varepsilon_{\text {out }}(1)$. Similarly, (51) reduces to $\tilde{\varepsilon}_{\text {out }}^{(e)}=\varepsilon_{\text {out }}(1)$, and therefore the secrecy outage probability will also be independent of $P_{e}$ when $\rho=0$. As expected, as $P_{e} \rightarrow 1$, $\tilde{\varepsilon}_{\text {out }}^{(e)}$ will approach $\varepsilon_{\text {out }}(1)$, which means that 


$$
\begin{aligned}
\tilde{\varepsilon}_{\text {out }}^{(e)} & =1-\int_{0}^{\infty} \int_{2^{R_{0}} \gamma_{w}+\left(2^{R_{0}}-1\right)}^{\infty} f^{(e)}\left(\tilde{\gamma}_{m}\right) f\left(\gamma_{w}\right) d \tilde{\gamma}_{m} d \gamma_{w} \\
& =(1-\chi)\left[1-\int_{0}^{\infty} \int_{2^{R_{0}} \gamma_{w}+\left(2^{R_{0}}-1\right)}^{\infty} f\left(\tilde{\gamma}_{m}\right) f\left(\gamma_{w}\right) d \tilde{\gamma}_{m} d \gamma_{w}\right]+\chi\left[1-\int_{0}^{\infty} \int_{2^{R_{0}} \gamma_{w}+\left(2^{R_{0}}-1\right)}^{\infty} p\left(\tilde{\gamma}_{m}\right) f\left(\gamma_{w}\right) d \tilde{\gamma}_{m} d \gamma_{w}\right] \\
& =(1-\chi) \tilde{\varepsilon}_{\text {out }}\left(N_{t}\right)+\chi \tilde{\varepsilon}_{\text {out }}(1)
\end{aligned}
$$

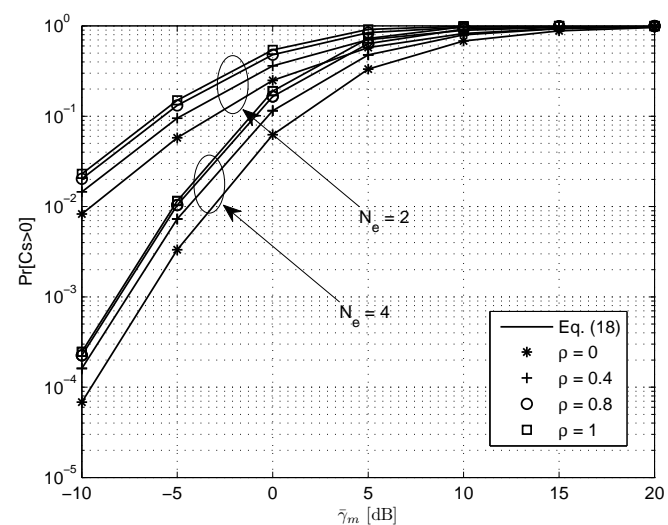

Fig. 2. The probability of non-zero secrecy rate versus the average SNR for the main channel $\bar{\gamma}_{m}$ with different correlation coefficients $\rho$ when $N_{t}=4$, $N_{r}=1, N_{e}=\{2,4\}$, and $\bar{\gamma}_{w}=0 \mathrm{~dB}$.

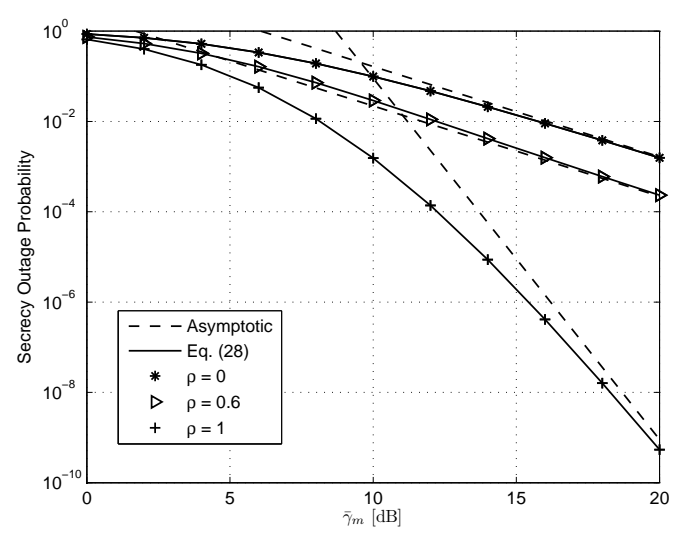

Fig. 3. Secrecy outage probability versus the average SNR for the main channel $\bar{\gamma}_{m}$ with different correlation coefficients $\rho$ when $N_{t}=4, N_{r}=2$, $N_{e}=2, R_{0}=1$ bits $/ \mathrm{s} / \mathrm{Hz}$, and $\bar{\gamma}_{w}=0 \mathrm{~dB}$.

the TAS/MRC-based scheme degenerates to the MRC-based scheme and only the MRC diversity gain can be obtained.

\section{Numerical Results}

\section{A. Secrecy performance}

In this section, we present numerical results to examine the effect of imperfect feedbacks on secrecy performance. In all the figures, the solid and dash lines represent numerical results using the closed-form expressions derived and the marks show the simulation results. As we can see, our analytical results all match perfectly with the simulations.

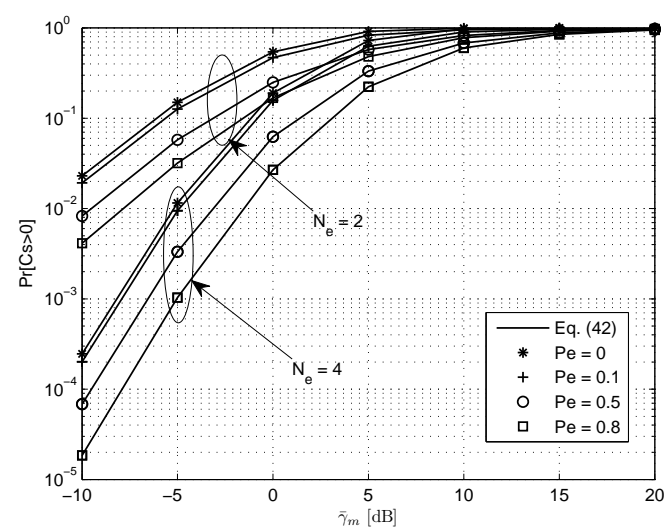

Fig. 4. The probability of non-zero secrecy rate versus the average SNR for the main channel $\bar{\gamma}_{m}$ with different $P_{e}$ when $N_{t}=4, N_{r}=1, N_{e}=\{2,4\}$, and $\bar{\gamma}_{w}=0 \mathrm{~dB}$.

Fig. 2 shows the probability of non-zero secrecy rate in (18) versus $\bar{\gamma}_{m}$. Results illustrate that for a fixed $\bar{\gamma}_{w}, \operatorname{Pr}\left\{R_{s}>0\right\}$ increases with $\bar{\gamma}_{m}$ and as $\bar{\gamma}_{m} \rightarrow \infty, \operatorname{Pr}\left\{R_{s}>0\right\}$ will approach one even when feedback experiences a time-delay. Moreover, $\operatorname{Pr}\left\{R_{s}>0\right\}$ will decrease with an increase in the number of the eavesdropper's antennas $N_{e}$. It is obvious that delayed feedbacks have detrimental effects on the secrecy performance, the larger the delay (i.e. smaller value of $\rho$ ), the smaller the probability of non-zero secrecy rate. We also observe that as $\rho$ increases, the secrecy performance can be enhanced. As shown in (20), a non-zero secrecy rate would exist even when the current channel is fully independent of the channel associated with the selected index, i.e., $\rho=0$.

In Fig. 3, results are provided for the secrecy outage probability using (28) and the asymptotic outage probability versus $\bar{\gamma}_{m}$. As can be seen, the asymptotic curves are very tight with the analytical ones at high SNRs and feedback delays have a significant impact on the secrecy outage probability. Results show that the loss of the secrecy outage performance due to feedback delays is especially obvious at high $\bar{\gamma}_{m}$, which causes the secrecy diversity loss. It is illustrated that when $\rho \neq 1$, as $\bar{\gamma}_{m} \rightarrow \infty$, the gain from TAS will eliminate and only the MRC diversity gain remains.

Figs. 4 and 5 study the effect of erroneous feedbacks on the secrecy performance using the analytical results (42) and (43), respectively. In the simulations, we assume that the feedback bits undergo a binary symmetric channel with error probability $P_{e}$, which introduces a bit error with probability $P_{e}$ and treats each bit independently. As shown in Fig. 4, the probability 


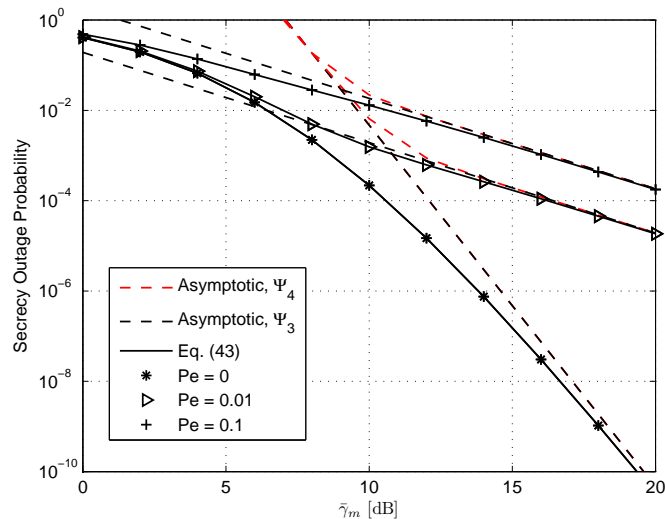

Fig. 5. Secrecy outage probability versus the average SNR for the main channel $\bar{\gamma}_{m}$ with different $P_{e}$ when $N_{t}=4, N_{r}=2, N_{e}=2, R_{0}=$ $0.5 \mathrm{bits} / \mathrm{s} / \mathrm{Hz}$, and $\bar{\gamma}_{w}=0 \mathrm{~dB}$.

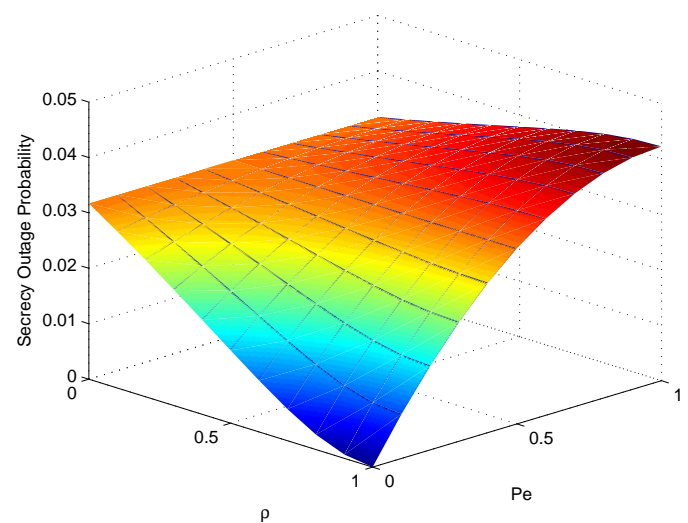

Fig. 6. Secrecy outage probability versus $P_{e}$ and $\rho$ when $N_{t}=4, N_{r}=1$, $N_{e}=2, R_{0}=0.5 \mathrm{bits} / \mathrm{s} / \mathrm{Hz}, \bar{\gamma}_{m}=20 \mathrm{~dB}$, and $\bar{\gamma}_{w}=0 \mathrm{~dB}$.

of non-zero secrecy rate reduces as $P_{e}$ increases. Although there exists erroneous feedbacks, $\operatorname{Pr}\left\{R_{s}>0\right\}$ will approach one as $\bar{\gamma}_{m} \rightarrow \infty$. The secrecy outage probability and the asymptotic outage probability versus $\bar{\gamma}_{m}$ are shown in Fig. 5 for various $P_{e}$. It can be seen that the erroneous feedbacks have a significant effect on the secrecy outage diversity, especially in high $\bar{\gamma}_{m}$. When $P_{e} \neq 0$, the diversity gain from TAS disappears and the receiver only obtains the MRC diversity gain. Also, the secrecy array gain $\Psi_{4}$ shows that the secrecy diversity order changes from $N_{t} N_{r}$ to $N_{r}$. It can be seen that as $\bar{\gamma}_{m} \rightarrow \infty$, the second term in (43) is more dominating in the high SNR regime; thus $\Psi_{4}$ approaches $\Psi_{3}$, as expected.

Fig. 6 presents the secrecy outage probability results (51) when both time-delayed feedbacks and erroneous feedbacks are considered. We observe that when $\rho=0$, i.e., random antenna selection, the secrecy outage probability is independent of $P_{e}$. A similar result can also be observed in Fig. 7, where $\bar{\gamma}_{w}=\bar{\gamma}_{m} / 10$. This corresponds to the case where the power at the transmitter increases, and $\bar{\gamma}_{w}$ increases with $\bar{\gamma}_{m}$. It can be seen that at very low SNR, the secrecy outage probability is close to one, which means that we cannot expect any secrecy. As expected, the time-delayed and erroneous feedbacks have significant effects on the secrecy outage performance.

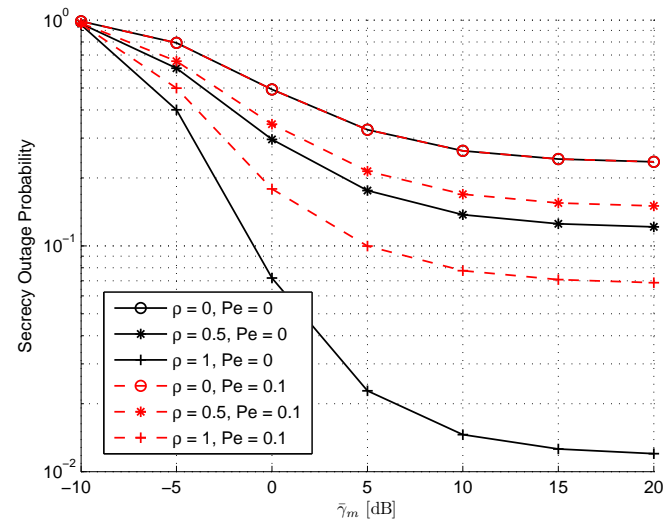

Fig. 7. Secrecy outage probability versus the average SNR for the main channel $\bar{\gamma}_{m}$ with different $P_{e}$ and $\rho$ when $N_{t}=4, N_{r}=1, N_{e}=2$, $R_{0}=0.5 \mathrm{bits} / \mathrm{s} / \mathrm{Hz}$, and $\bar{\gamma}_{w}=\bar{\gamma}_{m} / 10$.

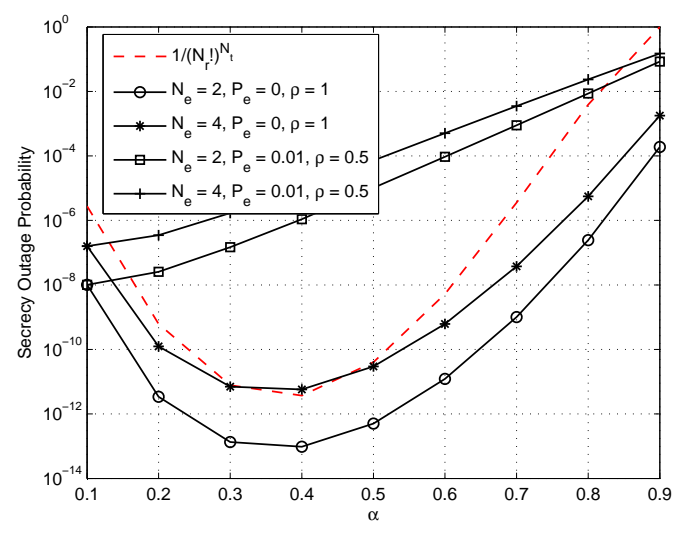

Fig. 8. The effect of $\alpha$ on secrecy outage probability with different $P_{e}$ and $\rho$ when $N_{\text {total }}=10, N_{e}=\{2,4\}, R_{0}=5 \mathrm{bits} / \mathrm{s} / \mathrm{Hz}, \bar{\gamma}_{m}=25 \mathrm{~dB}$, and $\bar{\gamma}_{w}=0 \mathrm{~dB}$.

Fig. 8 characterizes the effects of $\alpha=N_{t} / N_{\text {total }}$ on the secrecy outage probability with $N_{\text {total }}=10$. When $\rho \neq 1$ or $P_{e} \neq 0$, more antennas should be allocated to Bob, i.e., the best allocation to minimize the secrecy outage probability is $\alpha=0.1\left(N_{t}=1\right)$. However, when $\rho=1$ and $P_{e}=0$, the best $\alpha$ is between 0.3 and 0.4. As analyzed in Section III-C, we can adopt the function $1 /\left(N_{r} !\right)^{N_{t}}$ to replace the secrecy outage probability. The value of $1 /\left(N_{r} !\right)^{N_{t}}$ is also drawn in this figure. It can be found that the trends of the secrecy outage probability and the approximate function $1 /\left(N_{r} !\right)^{N_{t}}$ versus $\alpha$ match each other reasonably well.

\section{B. Comparison with other schemes}

For the typical passive eavesdropping scenario (in which the transmitter does not know the eavesdropper's channel), the masked beamforming method proposed in [9] and the codebook-based beamforming (CB) method proposed in [12] are two effective schemes to enhance secure communications. In this subsection, we compare the TAS-MRC with these schemes. For the sake of a fair comparison, we consider $N_{r}=1$, i.e., MISO wiretap channels. The transmitted signal 
for the masked beamforming is given as [9]

$$
\mathbf{x}=\sqrt{\lambda P} \mathbf{t} s+\sqrt{(1-\lambda) P} \mathbf{z}
$$

where $s$ is the data symbol with $\mathbb{E}\left\{|s|^{2}\right\}=1$, t represents the normalized beamforming vector with $\|\mathbf{t}\|=1$, and $\mathbf{z}$ is the AN vector with $\mathbf{R}_{z}=\mathbb{E}\left\{\mathbf{z z} \mathbf{z}^{\dagger}\right\}$ and trace $\left\{\mathbf{R}_{z}\right\}=1$. Also, $\lambda$ denotes the transmit power allocation parameter between data and $\mathrm{AN}, 0 \leq \lambda \leq 1$, and $P$ is the total transmit power.

According to [9], [10], the transmitter should choose the beamforming vector $\mathbf{t}$ as the principal eigenvector $\mathbf{t}_{1}$ which corresponds to the largest eigenvalue of $\mathbf{h h}^{\dagger}$, and the $\mathrm{AN}$ vector $\mathbf{z}$ can be expressed as a linear combination of the remaining $N_{t}-1$ eigenvectors, i.e, $\mathbf{z}$ lies in the nullspace of $\mathbf{h}^{\dagger}$. In addition, when $\lambda=1$, the masked beamforming scheme reduces to the naive beamforming (NB) scheme, i.e., no $\mathrm{AN}$ is used.

For $\mathrm{CB}$, the received signals at legitimate receiver and the eavesdropper can be expressed, respectively, as [12]

$$
\begin{aligned}
& y=\sqrt{P} \mathbf{h}^{\dagger} \mathbf{q} x+n_{b}, \\
& \mathbf{z}=\sqrt{P} \mathbf{G} \mathbf{q} x+\mathbf{n}_{e},
\end{aligned}
$$

where $\mathbf{q}=\arg \max _{\mathbf{c}_{i} \in \mathcal{C}}\left|\mathbf{h}^{\dagger} \mathbf{c}_{i}\right|$ is the selected codeword, and $\mathcal{C}=\left\{\mathbf{c}_{1}, \mathbf{c}_{2}, \cdots, \mathbf{c}_{N}\right\}$ is a pre-designed codebook of $N$ unit norm vectors, which is known to all parties (including the eavesdropper). Thus, the corresponding index of $\mathbf{q}$ should be fed back to the transmitter.

We apply two different codebooks for comparison. For $N=$ 4, we will design a codebook using the Generalized Lloyd Algorithm (GLA) (also known as LBG algorithm) presented in [32], where the minimum distance between different codes $\mathbf{w}_{i}$ and $\mathbf{w}_{j}$ is $\delta=\sqrt{1-\left|\mathbf{w}_{i}^{\dagger} \mathbf{w}_{j}\right|^{2}}=0.9999$. For $N=16$, we will use a Grassmannian codebook proposed in [33]. Thus, the feedbacks of 2 and 4 bits are necessary for the codebook sizes $N=4$ and $N=16$, respectively.

Fig. 9 compares the secrecy outage probability of the TASMRC scheme to other schemes, assuming $\bar{\gamma}_{m}=15 \mathrm{~dB}$ and $\bar{\gamma}_{w}=0 \mathrm{~dB}$. As we can see, when the normalized delay $f_{d} \tau$ is very small, the performance of 'MB' (masked beamforming) is much better than the others. However, when the channel is severely outdated, i.e., $f_{d} \tau$ is large, the performance of 'MB' significantly degrades because of noise leakage caused by imperfect CSI for the main channel. In addition, as $f_{d} \tau$ decreases, the performance of ' $\mathrm{MB}, \lambda=0.9$ ' is much better than ' $\mathrm{MB}, \lambda=0.5$ ' while ' $\mathrm{MB}, \lambda=0.5$ ' performs better when $f_{d} \tau$ is large. From the above simulation, we can obtain the following facts about 'MB':

1) If the main channel is perfectly known at the transmitter, AN can be made invisible to the legitimate receiver but only degrading the eavesdropper's reception. However, the imprecise knowledge of the main channel will cause noise leakage, thus significantly degrading the secrecy performance.

2) The performance of 'MB' largely depends on how well the power is allocated between the signal and the AN. As $f_{d} \tau$ decreases $(\rho \rightarrow 1)$, we should use more power to transmit the AN. However, most power should be used to transmit the signal if the CSI of the main channel is severely outdated.

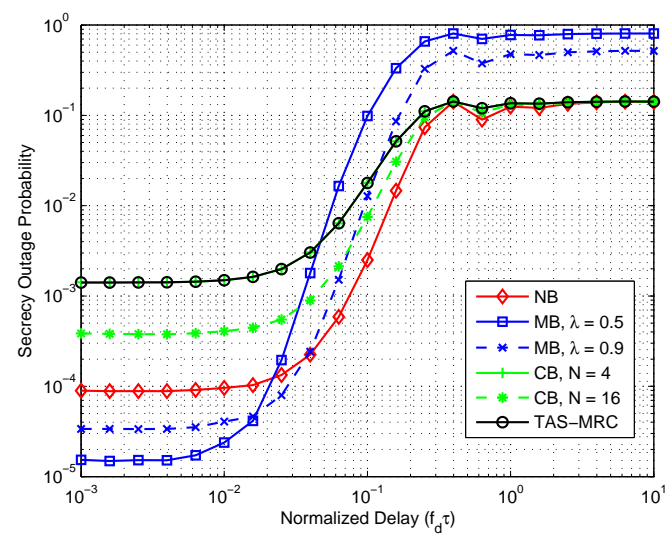

Fig. 9. Secrecy outage probability versus the normalized delay $f_{d} \tau$ when $N_{t}=4, N_{r}=1, N_{e}=2, R_{0}=1$ bits $/ \mathrm{s} / \mathrm{Hz}, \bar{\gamma}_{m}=15 \mathrm{~dB}$, and $\bar{\gamma}_{w}=0 \mathrm{~dB}$.

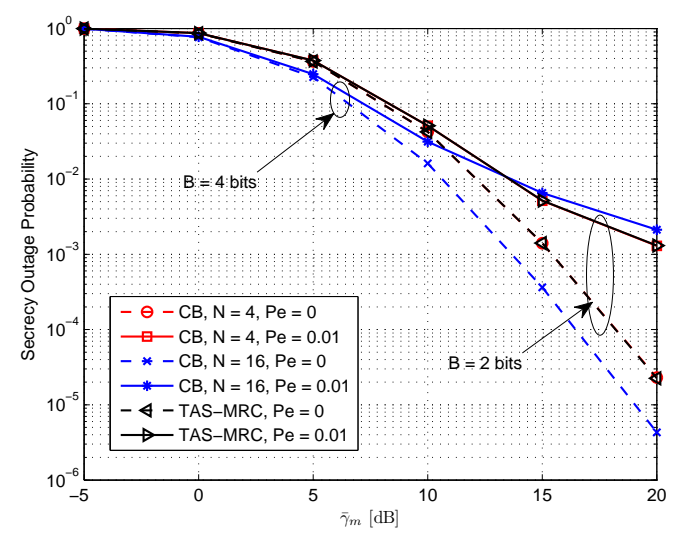

Fig. 10. Secrecy outage probability versus the average SNR for the main channel $\bar{\gamma}_{m}$ with different $P_{e}$ when $N_{t}=4, N_{r}=1, N_{e}=2, R_{0}=$ $1 \mathrm{bits} / \mathrm{s} / \mathrm{Hz}$, and $\bar{\gamma}_{w}=0 \mathrm{~dB}$.

From the figure we can also see that the ' $\mathrm{CB}, \mathrm{N}=16$ ' and 'NB' schemes outperform TAS-MRC. However, in the 'NB' scheme, Bob should transmit the complete CSI to Alice, requiring in theory an unlimited amount of feedback, while in the ' $\mathrm{CB}, \mathrm{N}=16$ ' scheme, the amount of feedback information is $B=\lceil\log N\rceil=4$ bits. The proposed TAS-MRC scheme only requires a $B=\left\lceil\log N_{t}\right\rceil=2$-bit feedback. In other words, when the feedback rate is fixed, the ' $\mathrm{NB}$ ' and ' $\mathrm{CB}$, $\mathrm{N}=16$ ' schemes would introduce a larger feedback delay compared with TAS-MRC.

Moreover, Fig. 10 compares the secrecy outage probability of the TAS-MRC scheme to the ' $\mathrm{CB}, \mathrm{N}=4$ ' and ' $\mathrm{CB}, \mathrm{N}=$ 16' schemes in presence of erroneous feedbacks. As shown, with perfect feedbacks, i.e., $P_{e}=0$, the secrecy performance of the ' $\mathrm{CB}, \mathrm{N}=16$ ' scheme is better than that of the TASMRC scheme. However, the ' $\mathrm{CB}, \mathrm{N}=16$ ' scheme requires higher feedback load, i.e., $B=4$ bits, thus causing higher feedback error than that of the TAS-MRC scheme according to $\varepsilon=1-\left(1-P_{e}\right)^{B}$. It can be found that when $P_{e}=0.01$, there exists a crossover point of $\bar{\gamma}_{m}$. Above this point, the TAS-MRC scheme outperforms the ' $\mathrm{CB}, \mathrm{N}=16$ ', which enhances the robustness to the feedback errors. In addition, from Figs. 9 and 10, we can find that the ' $\mathrm{CB}, \mathrm{N}=4$ ' 
TABLE I

COMPARISON WITH OTHER SCHEMES

\begin{tabular}{|c|c|c|c|c|c|c|c|}
\hline & & NB & $\mathrm{MB}, \lambda=0.5$ & $\mathrm{MB}, \lambda=0.9$ & $\mathrm{CB}, N=4$ & $\mathrm{CB}, N=16$ & TAS-MRC \\
\hline \multicolumn{2}{|r|}{ Number of RF Chains } & 4 & 4 & 4 & 4 & 4 & 1 \\
\hline \multicolumn{2}{|c|}{ Number of Feedback Bits ${ }^{*}$} & $\infty$ & $\infty$ & $\infty$ & 2 & 4 & 2 \\
\hline \multirow{6}{*}{$\mathrm{SOP}^{* *}$} & $f_{d} \tau=10^{-3}, \bar{\gamma}_{m}=15 \mathrm{~dB}$ & $8.96 \times 10^{-5}$ & $1.54 \times 10^{-5}$ & $3.38 \times 10^{-5}$ & $1.41 \times 10^{-3}$ & $3.85 \times 10^{-4}$ & $1.41 \times 10^{-3}$ \\
\hline & $f_{d} \tau=1, \bar{\gamma}_{m}=15 \mathrm{~dB}$ & 0.125 & 0.778 & 0.476 & 0.136 & 0.132 & 0.136 \\
\hline & $P_{e}=0.01, \bar{\gamma}_{m}=5 \mathrm{~dB}$ & - & - & - & 0.375 & 0.248 & 0.375 \\
\hline & $P_{e}=0.01, \bar{\gamma}_{m}=15 \mathrm{~dB}$ & - & - & - & $5.16 \times 10^{-3}$ & $6.52 \times 10^{-3}$ & $5.16 \times 10^{-3}$ \\
\hline & $P_{e}=0, \bar{\gamma}_{m}=5 \mathrm{~dB}$ & - & - & - & 0.366 & 0.226 & 0.366 \\
\hline & $P_{e}=0, \bar{\gamma}_{m}=15 \mathrm{~dB}$ & - & - & - & $1.41 \times 10^{-3}$ & $3.64 \times 10^{-4}$ & $1.41 \times 10^{-3}$ \\
\hline
\end{tabular}

${ }^{*}$ The number of feedback bits does not include the feedback of SNR value. ${ }^{* *}$, SOP' denotes the secrecy outage probability.

obtains the same secrecy performance as that of the TASMRC scheme. Although the feedback requirement is also $B=2$ bits for the ' $\mathrm{CB}, \mathrm{N}=4$ ' scheme, the TAS-MRC only requires a single $\mathrm{RF}$ chain, which effectively reduces the system's complexity. The overall comparison of these schemes in different scenarios is summarized in Table I, where $N_{t}=4$, $N_{r}=1, N_{e}=2, R_{0}=1 \mathrm{bits} / \mathrm{s} / \mathrm{Hz}$, and $\bar{\gamma}_{w}=0 \mathrm{~dB}$.

\section{CONCLUSIONS}

This paper studied the effects of imperfect feedback on the secrecy performance of MIMO wiretap channels with TAS at the transmitter and MRC at both the legitimate receiver and the eavesdropper. Time-delayed feedback and erroneous feedback were separately and jointly considered. We derived exact closed-form expressions for the probability of non-zero secrecy rate and secrecy outage probability. Our model provides useful insight into the secrecy performance in practical environments when time-delayed and/or erroneous feedbacks are present. Our analysis is general and encompasses the existing results as special cases. Specially, our asymptotic expressions revealed that imperfect feedbacks significantly degrade the secrecy performance and when the feedbacks are outdated and/or erroneous, the expected secrecy diversity order of $N_{t} N_{r}$ cannot be realized at high SNRs for the main channel. The secrecy diversity order is not affected by $N_{e}$.

\section{REFERENCES}

[1] A. D. Wyner, "The wire-tap channel," Bell Syst. Tech. J., vol. 54, no. 8, pp. 1355-1367, Oct. 1975.

[2] S. K. Leung-Yan-Cheong and M. E. Hellman, "The Gaussian wiretap channel," IEEE Trans. Inf. Theory, vol. 24, no. 4, pp. 451-456, Jul. 1978.

[3] I. Csiszár and J. Körner, "Broadcast channels with confidential messages," IEEE Trans. Inf. Theory, vol. 24, no. 3, pp. 339-348, May 1978.

[4] S. Shafiee and S. Ulukus, "Achievable rates in Gaussian MISO channels with secrecy constraints," in Proc. IEEE Int. Sym. Inf. Theory, pp. 24662470, Jun. 2007, Nice, France.

[5] A. Khisti and G. W. Wornell, "Secure transmission with multiple antennas-Part I: The MISOME wiretap channel," IEEE Trans. Inf. Theory, vol. 56, no. 7, pp. 3088-3104, Jul. 2010.

[6] T. Liu and S. Shamai, "A note on the secrecy capacity of the multiple antenna wiretap channel," IEEE Trans. Inf. Theory, vol. 55, no. 96, pp. 2547-2553, Nov. 2009.

[7] A. Khisti and G. W. Wornell, "Secure transmission with multiple antennas-Part II: The MIMOME wiretap channel," IEEE Trans. Inf. Theory, vol. 56, no. 11, pp. 5515-5532, Nov. 2010.

[8] F. Oggier and B. Hassibi, "The secrecy capacity of the MIMO wiretap channel," IEEE Trans. Inf. Theory, vol. 57, no. 8, pp. 4961-4972, Aug. 2011.
[9] S. Goel and R. Negi, "Guaranteeing secrecy using artificial noise," IEEE Trans. Wireless Commun., vol. 7, no. 6, pp. 2180-2189, Jun. 2008.

[10] J. Xiong, K. K. Wong, D. Ma, and J. Wei, "A closed-form power allocation for minimizing secrecy outage probability for MISO wiretap channels via masked beamforming," IEEE Commun. Lett., vol. 16, no. 9, pp. 1496-1499, Sep. 2012.

[11] A. Mukherjee and A. L. Swindlehurst, "Robust beamforming for security in MIMO wiretap channels with imperfect CSI," IEEE Trans. Signal Process., vol. 59, no. 1, pp. 351-361, Jan. 2011.

[12] S. Bashar, Z. Ding, and Y. G. Li, "On secrecy of codebook-based transmission beamforming under receriver limited feedback," IEEE Trans. Wireless Commun., vol. 10, no. 4, pp. 1212-1223, Apr. 2011.

[13] S. Sanayei and A. Nosratinia, "Antenna selection in MIMO systems," IEEE Commun. Mag., vol. 42, no. 10, pp. 68-73, 2004.

[14] T. Skinner and J. Cavers, "Selective diversity for Rayleigh fading channels with a feedback link," IEEE Trans. Commun., vol. 21, no. 2, pp. 117-126, Feb. 1973.

[15] S. Thoen, L. V. Perre, B. Gyselinckx, and M. Engels, "Performance analysis of combined transmit-SC/receive-MRC," IEEE Trans. Commun., vol. 49, no. 1, pp. 5-8, Jan. 2001.

[16] J. Tang and X. Zhang, "Transmit selection diversity with maximalratio combining for multicarrier DS-CDMA wireless networks over Nakagami-m fading channels," IEEE J. Select. Commun., vol. 24, no. 1, pp. 104-112, Jan. 2006.

[17] V. U. Prabhu and M. R. D. Rodrigues, "On wireless channels with $M$ antenna eavesdroppers: characterization of the outage probability and $\varepsilon$-outage secrecy capacity," IEEE Trans. Inf. Forensics Security, vol. 6, no. 3, pp. 853-860, Sep. 2011.

[18] F. M. He, H. Man, and W. Wang, "Maximal ratio diversity combining enhanced security," IEEE Commun. Lett., vol. 15, no. 5, pp. 509-511, May 2011.

[19] M. Z. I. Sarkar and T. Rarnarajah, "Enhancing security in correlated channel with maximal ratio combining diversity," IEEE Trans. Signal Process., vol. 60, no. 12, pp. 6745-6751, Dec. 2012.

[20] N. S. Ferdinand, D. B. da Costa, and M. Latva-aho, "Physical layer security in MIMO OSTBC line-of-sight wiretap channels with arbitrary transmit/receive antenna correlation," IEEE Wireless Commun. Lett., accepted for publication.

[21] H. Alves, R. D. Souza, M. Debbah, and M. Bennis, "Performance of transmit antenna selection physical layer security schemes," IEEE Signal Process. Lett., vol. 19, no. 6, pp. 372-375, Jun. 2012.

[22] N. Yang, P. L. Yeoh, M. Elkashlan, R. Schober, and I. B. Collings, "Transmit antenna selection for security enhancement in MIMO wiretap channels," IEEE Trans. Commun., vol. 61, no. 1, pp. 144-154, Jan. 2013.

[23] N. Yang, H. A. Suraweera, I. B. Collings, and C. Yuen, "Physical layer security of TAS/MRC with antenna correlation," IEEE Trans. Inf. Forensics Security, vol. 8, no. 1, pp. 254-259, Jan. 2013.

[24] N. Yang, P. L. Yeoh, M. Elkashlan, R. Schober, and J. Yuan, "MIMO wiretap channels: secure transmission using transmit antenna selection and receive generalized selection combining," IEEE Commun. Lett., vol. 17, no. 9, pp. 1754-1757, Sept. 2013.

[25] N. S. Ferdinand, D. B. da Costa, and M. Latva-aho, "Effects of outdated CSI on the secrecy performance of MISO wiretap channels with transmit antenna selection," IEEE Commun. Lett., vol. 17, no. 5, pp. 864-867, May. 2013.

[26] D. G. Brennan,"Linear diversity combining techniques," Proc. IRE, vol. 46, pp. 1075-1102, Jun. 1959 
[27] M. Bloch, J. Barros, M. Rodrigues, and S. McLaughlin, "Wireless information-theoretic security," IEEE Trans. Inf. Theory, vol. 54, no. 6, pp. 2515-2534, Jun. 2008.

[28] C. C. Tan and N. C. Beaulieu, "On first-order Markov modeling for the Rayleigh fading channel," IEEE Trans. Commun., vol. 48, no. 12, pp. 2032-2040, Dec. 2000

[29] M. S. Alouini and A. J. Goldsmith, "Adaptive modulation over Nakagami fading channels," Kluwer J. Wireless Commun., vol. 13, pp. 119-143, May 2000.

[30] I. S. Gradshteyn and I. M. Ryzhik, "Table of integrals, series and products," 7th ed. San Diego, CA: academic, 2007.

[31] H. A. David, "Order Statistics," 2nd ed. New York: Wiley, 1981.

[32] Y. Linde, A. Buzo, R. M. Gray, "An algorithm for vector quantizer design," IEEE Trans. Commun., vol. 28, no. 1, pp. 84-95, Jan. 1980.

[33] Available online: http://engineering.purdue.edu/ djlove/grass.html. 